\title{
Immune pathway upregulation and lower genomic instability distinguish EBV-positive nodal T/NK-cell lymphoma from ENKTL and PTCL-NOS
}

Cho Mar Myint Wai,,$^{1 *}$ Shangying Chen,,$^{2,}$ The Phyu, Shuangyi Fan, ${ }^{1}$ Sai Mun Leong, Wenning Zheng, ${ }^{3}$ Louis Ching Yi Low, ${ }^{1}$ Shoa-Nian Choo, Chi-Kuen Lee, Tae-Hoon Chung, ${ }^{3}$ Kenneth Hon Kim Ban, ${ }^{2}$ Soumita Ghosh, ${ }^{3}$ Stefanus Lie, ${ }^{3}$ Seiichi Kato, ${ }^{4,5}$ Shigeo Nakamura, ${ }^{4}$ Emiko Takahashi, ${ }^{6}$ Young-Hyeh Ko, Joseph D. Khoury, ${ }^{8}$ Shih-Sung Chuang, ${ }^{9}$ Rex K.H. Au-Yeung, ${ }^{10}$ Soo-Yong Tan,, 11 Soon-Thye Lim, ${ }^{12}$ Choon-Kiat Ong, ${ }^{13-15}$ Yong-Howe Ho, ${ }^{16}$ Li Mei Poon, ${ }^{17}$ Sanjay de Mel, ${ }^{17}$ Anand D. Jeyasekharan, ${ }^{3}$ Wee-Joo Chng, ${ }^{3,7,18}$ Franziska Otto, ${ }^{19}$ Leticia Quintanilla-Martinez, ${ }^{19}$ Federica Zanardi, ${ }^{20}$ Fabio lannelli, ${ }^{20}$ Claudio Tripodo, ${ }^{21}$ Jason J. Pitt ${ }^{3}$ and Siok-Bian $\mathrm{Ng}^{1,3,11}$

'Department of Pathology, Yong Loo Lin School of Medicine, National University of Singapore, Singapore; ${ }^{2}$ Department of Biochemistry, Yong Loo Lin School of Medicine, National University of Singapore, Singapore; ${ }^{3}$ Cancer Science Institute of Singapore, National University of Singapore, Singapore; ${ }^{4}$ Department of Pathology and Laboratory Medicine, Nagoya University Hospital, Nagoya, Japan; ${ }^{5}$ Department of Pathology and Molecular Diagnostics, Aichi Cancer Center Hospital, Nagoya, Japan; ${ }^{6}$ Department of Pathology, Aichi Medical University Hospital, Nagakute, Japan; ${ }^{7}$ Department of Pathology, Samsung Medical Center, Sungkyunkwan University, Seoul, Korea; ${ }^{8}$ Department of Hematopathology, The University of Texas MD Anderson Cancer Center, Houston, TX, USA; ${ }^{9}$ Department of Pathology, Chi-Mei Medical Center, Tainan, Taiwan; ${ }^{10}$ Department of Pathology, Queen Mary Hospital, The University of Hong Kong, Hong Kong SAR, China; ${ }^{11}$ Department of Pathology, National University Hospital, National University Health System, Singapore; ${ }^{12}$ Lymphoma Genomic Translational Research Laboratory, National Cancer Center Singapore, Singapore; Division of Medical Oncology, National Cancer Center Singapore, Singapore; ${ }^{13}$ Lymphoma Genomic Translational Research Laboratory, Division of Medical Oncology, National Cancer Centre Singapore, Singapore; ${ }^{14}$ Duke-NUS Medical School, Singapore; ${ }^{15} \mathrm{Genome} \mathrm{Institute} \mathrm{of}$ Singapore, A*STAR (Agency for Science, Technology and Research), Singapore; ${ }^{16}$ Department of Pathology, Tan Tock Seng Hospital, Singapore; ${ }^{17}$ Department of Hematology-Oncology, National University Cancer Institute Singapore, National University Hospital, National University Health System, Singapore; ${ }^{18}$ Department of Medicine, Yong Loo Lin School of Medicine, National University of Singapore, Singapore; ${ }^{19}$ Institute of Pathology and Neuropathology, Eberhard Karls University of Tübingen and Comprehensive Cancer Center, Tübingen University Hospital, Tübingen, Germany; ${ }^{20}$ Bioinformatics Unit, IFOM - the FIRC Institute of Molecular Oncology, Milan, Italy and ${ }^{21}$ Tumor Immunology Unit, University of Palermo School of Medicine, Palermo, Italy

\author{
Correspondence: \\ Siok-Bian Ng (lead contact) \\ patnsb@nus.edu.sg \\ Jason J. Pitt \\ jason.j.pitt@nus.edu.sg \\ Received: September 9, 2021. \\ Accepted: $\quad$ January 4, 2022. \\ Prepublished: January 13, 2022. \\ https://doi.org/10.3324/haematol.2021.280003 \\ ๑2022 Ferrata Storti Foundation \\ Haematologica material is published under \\ a CC-BY-NC license @@
}

${ }^{*}$ CMMW and SC contributed equally as co-first authors.

\begin{abstract}
Primary Epstein-Barr virus (EBV)-positive nodal T/NK-cell lymphoma (PTCL-EBV) is a poorly understood disease which shows features resembling extranodal NK/T-cell lymphoma (ENKTL) and is currently not recognized as a distinct entity but categorized as a variant of primary T-cell lymphoma not otherwise specified (PTCL-NOS). Herein, we analyzed copynumber aberrations $(n=77)$ with a focus on global measures of genomic instability and homologous recombination deficiency and performed gene expression $(n=84)$ and EBV miRNA expression $(n=24)$ profiling as well as targeted mutational analysis $(n=16)$ to further characterize PTCL-EBV in relation to ENKTL and PTCL-NOS. Multivariate analysis revealed that patients with PTCL-EBV had a significantly worse outcome compared to patients with PTCL-NOS $(P=0.002)$ but not to those with ENKTL. Remarkably, PTCL-EBV exhibited significantly lower genomic instability and homologous recombination deficiency scores compared to ENKTL and PTCL-NOS. Gene set enrichment analysis revealed that many immune-related pathways, interferon $\alpha / \gamma$ response, and IL6_JAK_STAT3 signaling were significantly upregulated in PTCLEBV and correlated with lower genomic instability scores. We also identified that NFKB-associated genes, BIRC3, NFKB1 (P50) and CD27, and their proteins are upregulated in PTCL-EBV. Most PTCL-EBV demonstrated a type 2 EBV latency pattern and, strikingly, exhibited downregulated expression of most EBV miRNA compared to ENKTL and their target
\end{abstract}


genes were also enriched in immune-related pathways. PTCL-EBV also showed frequent mutations of TET2, PIK3CD and STAT3, and are characterized by microsatellite stability. Overall, poor outcome, low genomic instability, upregulation of immune pathways and downregulation of EBV miRNA are distinctive features of PTCL-EBV. Our data support the concept that PTCL-EBV could be considered as a distinct entity, provide novel insights into the pathogenesis of the disease and offer potential new therapeutic targets for this tumor.

\section{Introduction}

Primary nodal Epstein-Barr virus (EBV)-positive T/NK-cell lymphoma is an uncommon group of peripheral T-cell lymphomas (PTCL) that presents primarily with lymph node disease but may involve a limited number of extranodal organs. ${ }^{1}$ While the majority of PTCL-EBV are derived from T cells, a minority are bona fide NK-cell lymphomas. They show significant overlap with extranodal NK/T-cell lymphoma, nasal type (ENKTL) as both tumors are associated with EBV and characterized by cytotoxic T- or NKcell proliferation. EBV-positive nodal T/NK-cell lymphomas are more common in the elderly, usually demonstrate a monomorphic growth pattern and lack angiodestruction and prominent necrosis. ${ }^{2,3} \mathrm{~A}$ few reports have described clinicopathological features distinct from those of ENKTL, including the lack of nasal involvement, frequent T-cell origin, and a $\mathrm{CD}^{+} / \mathrm{CD}^{-} 6^{-}$phenotype..$^{2,4}$

The 2017 World Health Organization (WHO) lymphoma classification recommends that EBV-positive nodal T/NKcell lymphoma be considered as an EBV-positive variant of PTCL, not otherwise specified (PTCL-NOS) as data on this disease are limited, and it is currently unclear whether this group of lymphomas represents a distinct entity. ${ }^{5}$ The molecular biology of EBV-positive nodal T/NKcell lymphoma, henceforth referred to as an EBV-positive variant of PTCL (PTCL-EBV), and its relationship with ENKTL and PTCL-NOS remains poorly understood - mainly due to the rarity of these tumors and lack of tissue availability.

Genomic instability (GI) is a hallmark of cancer and refers to the propensity of cells to accumulate a variety of DNA alterations. These alterations, a subset of which provides a selective growth advantage instrumental for tumorigenesis and progression, may delineate different profiles in entities sharing phenotypic or transcriptional features. ${ }^{6}$ Importantly, the broad characteristics of Gl, as measured by scores, have prognostic and management implications, specifically with regard to the choice of therapeutic agents. $^{?}$

Given the rarity of PTCL-EBV, scarcity of frozen samples and lack of available cell lines, we leveraged formalinfixed paraffin-embedded tissues. Using gene expression profiling and testing of copy number aberrations, we previously compared PTCL-EBV to ENKTL and showed that PTCL-EBV is characterized by PD-L1 upregulation, expression of $\mathrm{T}$-cell related genes and frequent loss of 14q11.2, which correlates with loss of TCRA loci and Tcell origin. ${ }^{4,8}$ For the current study, we expanded the disease types and included PTCL-NOS in the comparison, and performed a comprehensive suite of analyses including EBV miRNA expression, custom gene panel sequencing and copy number aberration analysis focusing on global measures of GI. We further re-profiled the gene expression signatures using an improved microarray on our cases, including those studied in the previous work. ${ }^{4}$ Interestingly, we demonstrated that PTCL-EBV displays a remarkably lower degree of GI despite its more aggressive outcome among the three diseases and is characterized by upregulation of immune-related pathways and lower expression of EBV miRNA compared to ENKTL and PTCL-NOS. These novel findings not only offer insights into the pathogenesis of PTCL-EBV and raise considerations regarding its treatment, but also support the proposal that PTCL-EBV is a distinct entity in the WHO classification.

\section{Methods}

\section{Study cohort}

Cases from multiple institutions were reviewed by two hematopathologists to confirm the diagnosis of ENKTL $(n=89)$, PTCL-EBV $(n=25)$ and PTCL-NOS $(n=36)$ based on the 2017 WHO lymphoma classification. ${ }^{5}$ PTCL-EBV shows a similar phenotype as ENKTL but, unlike ENKTL, (i) patients present primarily with nodal disease where the bulk of tumor is localized; (ii) nasal involvement is lacking; (iii) it often shows a $\mathrm{CD} 8^{+} / \mathrm{CD} 56^{-}$phenotype; and (iv) it is often of T-cell origin (Online Supplementary Table S1). Systemic and cutaneous EBV-positive T/NK lymphoproliferative diseases occurring in children were excluded. The diagnosis of PTCL-NOS was established when other specific subtypes of PTCL had been excluded. Cytotoxicity was defined as the expression of at least one cytotoxic marker (TIA1, granzyme B). Clinical data including age, sex, disease type, stage, International Prognostic Index (IPI) score, expression of CD4, CD8 and CD56, T or NK lineage, treatment and overall survival were obtained (Online Supplementary Table $S 2 A, B)$. This study was approved by the National Healthcare Group Domain Specific Review Board B (2009/00212).

\section{Copy number aberration analysis}

An OncoScan $®$ FFPE assay was performed on 34 ENKTL, 
14 PTCL-EBV and 29 PTCL-NOS cases as previously published. ${ }^{9}$ Copy number aberrations were analyzed using OncoScan ${ }^{\circledR}$ Console (v1.3) software (ThermoFisher Scientific, Waltham, MA, USA). Segmentation results were analyzed using GISTIC (v2.0.22) ${ }^{10}$ with the GENCODE hg19 build. GI and homologous recombination deficiency (HRD) scores were calculated according to published methods. ${ }^{11}$

\section{Gene expression profiling}

Gene expression profiling was performed on 35 ENKTL, 23 PTCL-EBV, and 26 PTCL-NOS cases using a GeneChip ${ }^{\circledR}$ Clariom D Assay (Human) array and the data were analyzed as described previously. ${ }^{12}$ Gene expression profiling (GSE160119) and copy number aberration (GSE160118) raw data were deposited in the Gene Expression Omnibus (GEO).

\section{Polymerase chain reaction analysis of EBV miRNA and EBV latency}

Quantitative reverse transcription polymerase chain reaction (RT-qPCR) analysis of miRNA expression was performed using IDEAL miRNA qPCR assays (MiRXES, Singapore) ( $n=24)$ according to the manufacturer's instructions on a QuantStudio ${ }^{\mathrm{TM}} 5$ System (ThermoFisher Scientific, Waltham, MA, USA). RT-qPCR of EBV genes was performed to confirm EBV latency in PTCL-EBV and ENKTL.

\section{Fluorescence in situ hybridization and multiplex immunofluorescence}

Fluorescence in situ hybridization (FISH), multiplex immunofluorescence and multispectral imaging were performed as previously described. ${ }^{4}$

\section{Mutational analysis targeted next-generation sequencing}

Targeted mutation analysis was performed by next-generation sequencing (Ion GeneStudio S5 prime, Thermo Fisher Scientific, Waltham, MA, USA) using an Ampliseq customized T/NK-lymphoid panel comprising 35 genes recurrently mutated in ENKTL and PTCL-NOS (Online Supplementary Table S3A) and the 484-gene NovoPMTM 2.0 panel (Online Supplementary Table S3B) (total 500 genes including 19 common genes).

\section{Statistical methods}

Overall survival was investigated using Kaplan-Meier nonparametric survival analysis and log-rank tests. The effects of disease type, age, sex and disease stage were analyzed using unadjusted univariate and multivariate Cox proportional hazard models. Analyses were performed in $\mathrm{R}$ with the "survival" (v0.1.2) and "survminer" (v0.4.6) packages. Gene expression profiling analysis was performed in $\mathrm{R}$ with the "limma" package (v3.40.6). Unsupervised hier- archical clustering was performed using Spearman distance and Ward.D2 linkage. All additional statistical tests were performed in $\mathrm{R}$.

The diagnostic criteria for the diseases, T/NK lineage assignment, detailed descriptions of study cohorts, experimental methods, data analysis and data accession are provided in the Online Supplementary Materials.

\section{Results}

\section{Differences in clinical and survival variables among the disease groups}

The salient clinicopathological and immunohistochemical features of ENKTL, PTCL-EBV and PTCL-NOS are summarized in Table 1A and Online Supplementary Table S2A, B. All 25 patients with PTCL-EBV, comprising 14 Japanese, eight Chinese, two Korean and one Bangladeshi person, presented with nodal disease and the bulk of disease involved lymph nodes. Phenotypically, PTCL-EBV was often positive for CD8 $(17 / 25,68 \%)$, cytotoxic markers (100\%) and TCRB (12/25, 48\%). CD56 was commonly negative $(18 / 24,75 \%)$ and none of the cases tested expressed TCRG. Four of 23 (17\%) cases tested expressed CD4, of which one was positive for CXCL13 and CD10 (focal). Most of the cases of PTCL-EBV were of T-cell origin (20/23, $87 \%$ ), in accordance with previous reports ${ }^{3,13-15}$ (Figure 1A). With regards to treatment, the patients in all three groups from different institutions were treated with a heterogeneous combination of chemotherapy regimens (Online Supplementary Table S2A). This is understandable as these are rare lymphomas lacking standardized and effective treatment. Two out of 15 PTCL-EBV patients with known treatment data were treated with SMILE therapy. One died 3.5 months after diagnosis and the other died 12.6 months after diagnosis.

Despite the treatment heterogeneity, our results revealed that patients with PTCL-EBV had a significantly shorter median overall survival (4.6 months) compared to those with ENKTL (14.7 months, $P=0.001$ ) and PTCL-NOS (26 months, $P=0.007$ ). (Figure 1B; Table 1). Univariate analysis identified advanced disease stage (stage 4) $(P<0.001)$ and older age $(P=0.001)$ as significantly associated with poor prognosis. Compared to patients with PTCL-EBV, patients with ENKTL had a $61 \%$ lower risk of death $(H R=0.39,95 \%$ Cl: 0.22-0.69, $P=0.001$ ), while those with PTCL-NOS had a $59 \%$ lower risk ( $\mathrm{HR}=0.41,95 \% \mathrm{Cl}: 0.21-0.80, P=0.009$ ). After adjusting for disease stage, sex and age, PTCL-NOS remained significantly less aggressive compared to PTCLEBV (HR=0.3, 95\% Cl: 0.14-0.65, $P=0.002$ ) (Table 2). A Cox proportional-hazards model was also performed and after controlling for lineage, patients with PTCL-EBV showed significantly worse outcome than those with ENKTL $(\mathrm{HR}=2.34,95 \% \mathrm{Cl}: 1.05-5.24, P=0.038)$, indicating that the 
A

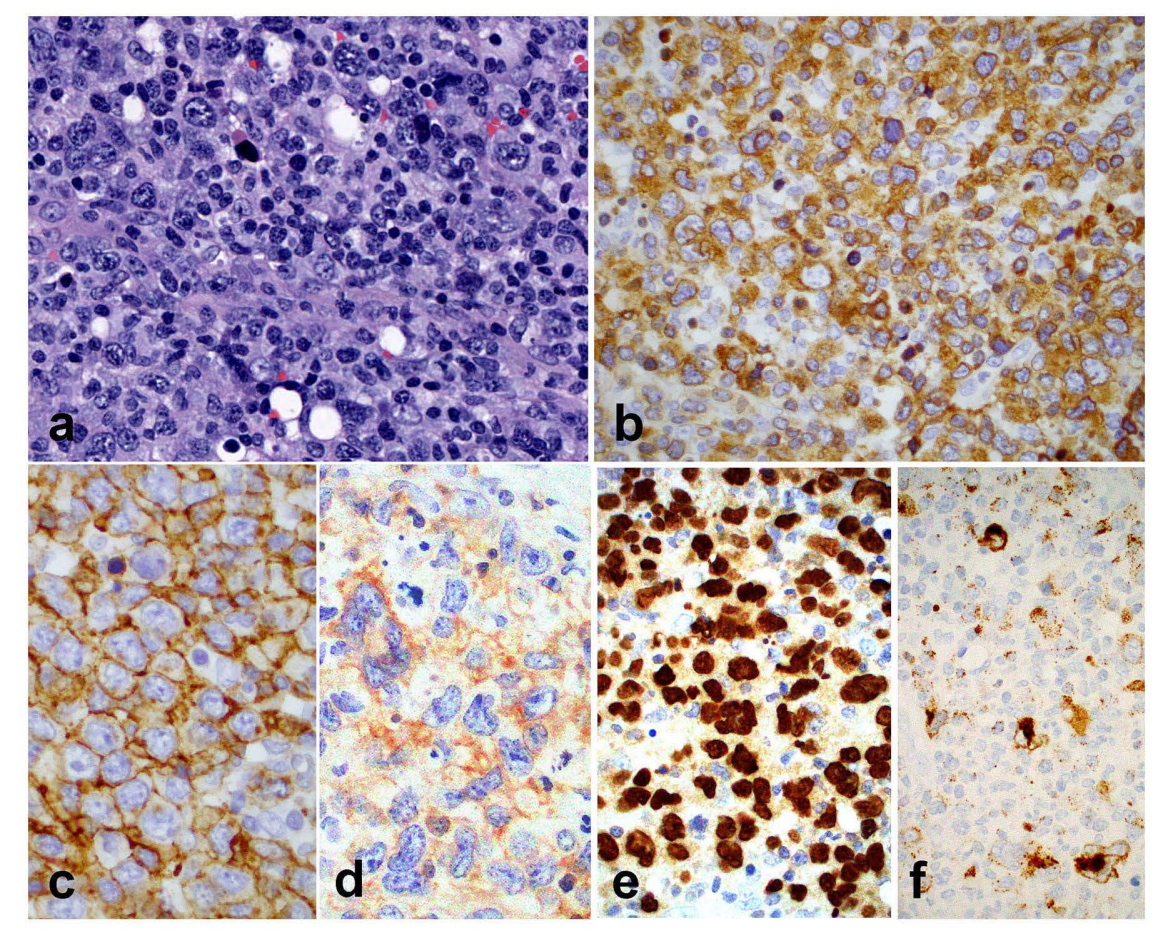

B

B Type + ENKTL + PTCL-EBV + PTCL-NOS

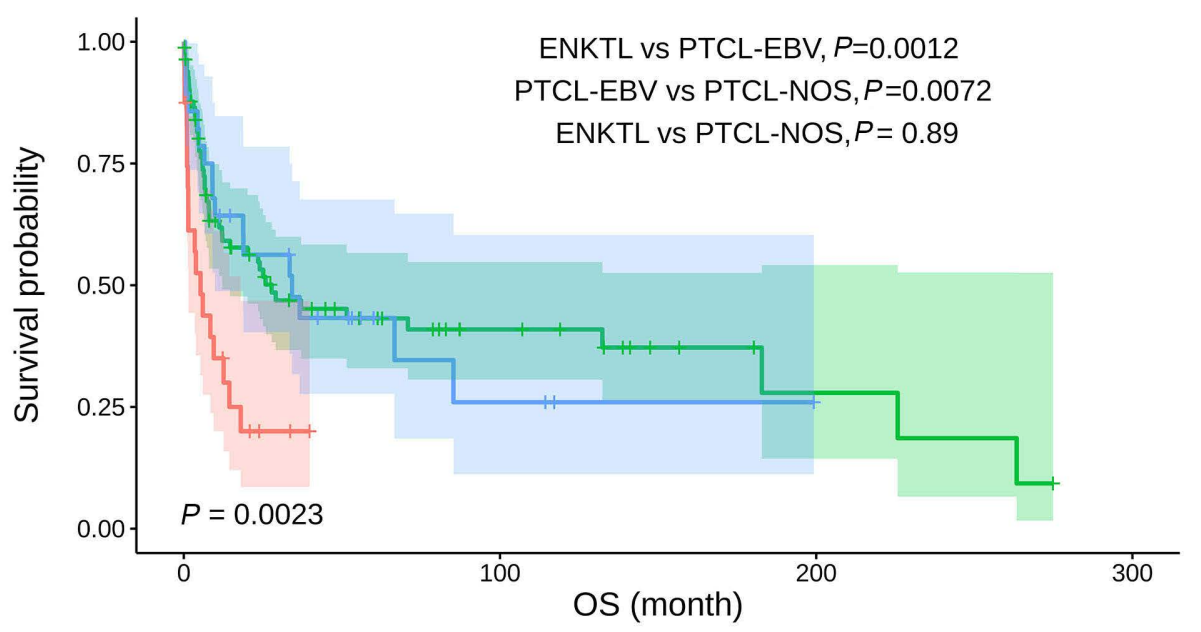

Number at risk

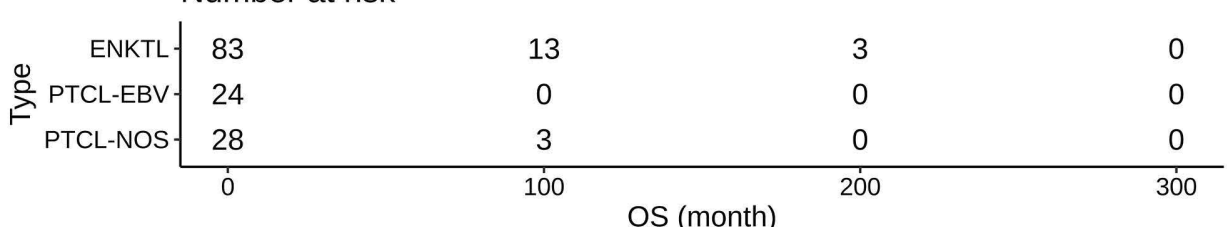

Figure 1. Morphological features and survival of PTCL-EBV patients compared to those with ENKTL and PTCL-NOS. (A) Representative images of PTCL-EBV. The tumor cells are large and pleomorphic (a, Hematoxylin \& eosin, original magnification $\mathrm{x} 400$ ). They are positive for CD3 (b, original magnification $\mathrm{x400),} \mathrm{CD8} \mathrm{(c,} \mathrm{original} \mathrm{magnification} \times 600)$, $T$-cell receptor, beta (TCR $)$ (d, original magnification $\times 600)$, EBER (e, original magnification $\times 400$ ) and granzyme $B(f$, original magnification $\times 400)$. The positive expression for TCR $\beta$ indicates a T-cell origin. (B) Kaplan-Meier survival curve depicting overall survival of three disease groups. Patients in the PTCL-EBV group had significantly shorter overall survival compared to those in the ENKTL and PTCL-NOS groups.

Table 1. Clinicopathological features and gene expression profiling in patients with ENKTL, PTCL-EBV and PTCL-NOS.

\begin{tabular}{|c|c|c|c|c|}
\hline Parameters & ENKTL $(\mathrm{N}=89)$ & PTCL-EBV (N=25) & PTCL-NOS $(\mathrm{N}=36)$ & $\boldsymbol{P}$ \\
\hline $\begin{array}{l}\text { Median age in years } \\
\text { (range, SD) }\end{array}$ & $49(17-82,16.20)$ & $59(32-89,14.43)$ & $62(11-95,18.22)$ & $0.002^{*}$ \\
\hline Female/male, $\mathrm{N}$ & $27 / 62$ & $5 / 20$ & $13 / 23$ & $0.399^{\dagger}$ \\
\hline $\mathrm{COO}, \mathrm{T} / \mathrm{NK} /$ indeterminate, $\mathrm{N}$ & $16 / 57 / 16$ & $20 / 3 / 2$ & $36 / 0 / 0$ & $<0.001^{\dagger}$ \\
\hline Stage $^{\ddagger}, 1 \& 2 / 3 \& 4, N$ & $52 / 29$ & $3 / 20$ & $4 / 22$ & $<0.001^{\dagger}$ \\
\hline $\begin{array}{l}\text { IPI score } \neq \\
\text { low/intermediate/high, N }\end{array}$ & $37 / 24 / 8$ & $3 / 11 / 6$ & $1 / 6 / 5$ & $0.001^{\dagger}$ \\
\hline Median survival ${ }^{\ddagger}$ in months & 14.7 & 4.6 & 26.0 & $0.002^{\dagger}$ \\
\hline CD56 ${ }^{\ddagger}$ positive/negative, $N$ & $66 / 20$ & $6 / 18$ & $1 / 25$ & $<0.001^{\dagger}$ \\
\hline $\mathrm{CD}^{\ddagger}{ }^{\ddagger}$ positive/negative, $\mathrm{N}$ & $11 / 64$ & $17 / 8$ & $11 / 25$ & $<0.001^{\dagger}$ \\
\hline TCR $\beta^{\ddagger}$ positive/negative, $N$ & $9 / 51$ & $12 / 13$ & $12 / 6$ & $<0.001^{\dagger}$ \\
\hline TCR $\gamma^{\ddagger}$ positive/negative, $\mathrm{N}$ & $2 / 54$ & $0 / 14$ & $6 / 12$ & $0.002^{\dagger}$ \\
\hline
\end{tabular}

${ }^{*}$ Kruskal-Wallis test, ${ }^{\dagger}$ chi-square test, ${ }^{\ddagger}$ only cases with available data were included for analysis, ${ }^{\S}$ log-rank test; SD: standard deviation; COO, cell of origin; IPI: International Prognostic Index. 
Table 2. Univariate and multivariate analyses for overall survival in the ENKTL, PTCL-EBV and PTCL-NOS groups.

\begin{tabular}{|c|c|c|c|c|c|c|c|}
\hline \multirow{2}{*}{ Variable } & \multirow[t]{2}{*}{ Category } & \multicolumn{3}{|c|}{ Univariate } & \multicolumn{3}{|c|}{ Multivariate } \\
\hline & & HR & Cl (95\%) & $\boldsymbol{P}$ & HR & Cl (95\%) & $\boldsymbol{P}$ \\
\hline \multirow[t]{3}{*}{ Disease type } & ENKTL & 0.39 & $0.22-0.69$ & 0.001 & 0.65 & $0.34-1.26$ & 0.2 \\
\hline & PTLC-EBV & 1 & Ref & & 1 & Ref & \\
\hline & PTCL-NOS & 0.41 & $0.21-0.80$ & 0.009 & 0.3 & $0.14-0.65$ & 0.002 \\
\hline \multirow[t]{4}{*}{ Stage } & 1 & 1 & Ref & & 1 & Ref & \\
\hline & 2 & 1.33 & $0.54-3.22$ & 0.50 & 1.5 & $0.61-3.67$ & 0.4 \\
\hline & 3 & 2.46 & $1.00-6.07$ & 0.051 & 1.56 & $0.51-4.81$ & 0.4 \\
\hline & 4 & 4.18 & $2.31-7.57$ & $<0.001$ & 4.56 & $2.43-8.54$ & $<0.001$ \\
\hline \multirow[t]{2}{*}{ Sex } & Female & 1 & Ref & & 1 & Ref & \\
\hline & Male & 1.23 & $0.76-1.99$ & 0.40 & 1.38 & $0.82-2.32$ & 0.2 \\
\hline Age & & 1.02 & $1.01-1.04$ & 0.001 & 1.02 & $1.01-1.04$ & 0.006 \\
\hline \multirow[t]{3}{*}{ IPI score } & Low & 1 & Ref & & & & \\
\hline & Intermediate & 5.16 & $2.32-11.5$ & $<0.001$ & & & \\
\hline & High & 8.33 & $3.48-19.9$ & $<0.001$ & & & \\
\hline
\end{tabular}

HR: hazard ratio; Cl: confidence interval; Ref: reference; IPI: International Prognostic Index.

worse outcome of PTCL-EBV patients compared to ENKTL patients is independent of lineage. Interestingly, there was no longer a significant difference in the survival outcome between ENKTL and PTCL-EBV patients, suggesting that the worse outcome of those with PTCL-EBV could be ascribed to older age and more advanced disease stage at diagnosis.

\section{Differences in focal copy number aberrations among disease groups}

In order to identify differences in copy number profiles of the three diseases, we analyzed copy number aberrations of patients with ENKTL $(n=34)$, PTCL-EBV $(n=14)$ and PTCLNOS $(n=29)$ and identified recurrent aberrations $(q<0.25)$ with their putative target genes across all samples (Figure $2 A)$. Previously, ${ }^{4}$ comparing ENKTL $(n=29)$ and PTCL-EBV $(n=12)$, we noted differences in $14 q 11.2$ and were able to reproduce those here. However, with the addition of five and two new ENKTL and PTCL-EBV samples, respectively - as well as a cohort of PTCL-NOS - we were able to identify multiple unreported differences in focal copy number aberration rates across these groups.

Of the 11 recurrently gained regions, 3p14.1, 6p22.3, 6p22.1 and 17q21.33 occurred at significantly different frequencies across disease groups ( $P<0.05, \chi^{2}$ test) (Online Supplementary Table S4). Gain of 3p14.1 was found in $14.3 \%$ of PTCLEBV cases compared to $5.9 \%$ and $76.0 \%$ of ENKTL and PTCL-NOS cases, respectively $(P<0.001)$. Gain of $6 \mathrm{p} 22.3$ was observed more frequently in ENKTL (20.6\%) and PTCL-NOS (58.6\%) than in PTCL-EBV $(7.1 \%)(P=0.005)$. Gain of 6p22.1 was observed in $21.4 \%$ of PTCL-EBV cases compared to $8.8 \%$ of ENKTL and $58.6 \%$ of PTCL-NOS cases
$(P=0.001)$. Of the nine recurrent losses, only $14 q 11.2$ occurred at significantly different frequencies among the disease groups (Online Supplementary Table S5). As expected, loss of $14 q 11.2$ was observed in $100 \%$ of PTCL-EBV cases, ${ }^{4}$ whereas it was only found in $20.6 \%$ and $58.6 \%$ of ENKTL and PTCL-NOS, respectively $(P=0.001)$. It is unclear whether the high frequency of $14 q 11.2$ loss in PTCL-EBV is a result of additional critical driver events occurring at the TCRA locus, on top of monoclonal TCRA gene rearrangement, or related to preferential TCR usage in PTCL-EBV due to possible underlying immune perturbations and antigen selection that predispose to malignancy. ${ }^{16}$ The $14 q 11.2$ loss in PTCL-NOS is slightly low, which may be a result of coverage bias related to the probe design of the Oncoscan assay or a lack of TCRA rearrangement in some PTCL-NOS cases. ${ }^{8}$ The top two recurrent copy-number gains (3p14.1 and 6p22.1) were subsequently validated by FISH (Online Supplementary Figure S1 and Online Supplementary Table S6).

\section{PTCL-EBV exhibits lower genomic complexity than other disease groups}

We further compared the genome-wide levels of copy number aberrations across the three disease types (Figure 2B, Online Supplementary Figure S2). Analysis of copy number burden (segment counts) revealed that PTCL-EBV exhibited significantly fewer segments than ENKTL $(P=0.016$, Mann-Whitney $U$ test $)$ and PTCL-NOS $(P<0.001)$ (Figure 2C, left panel). While copy number segment differences between ENKTL and PTCL-EBV appeared primarily driven by gains (Figure 2C, middle panel), PTCL-NOS demonstrated increased losses compared to other groups 
A

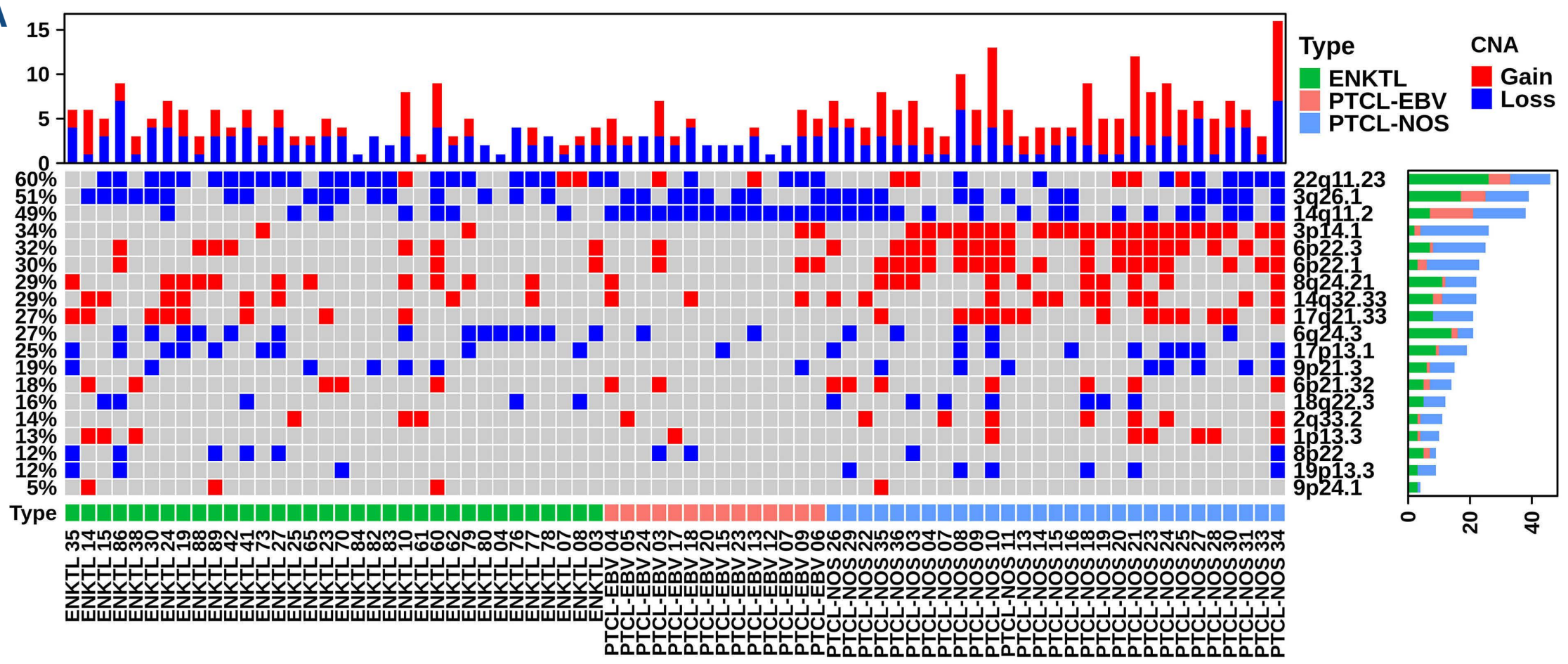

B
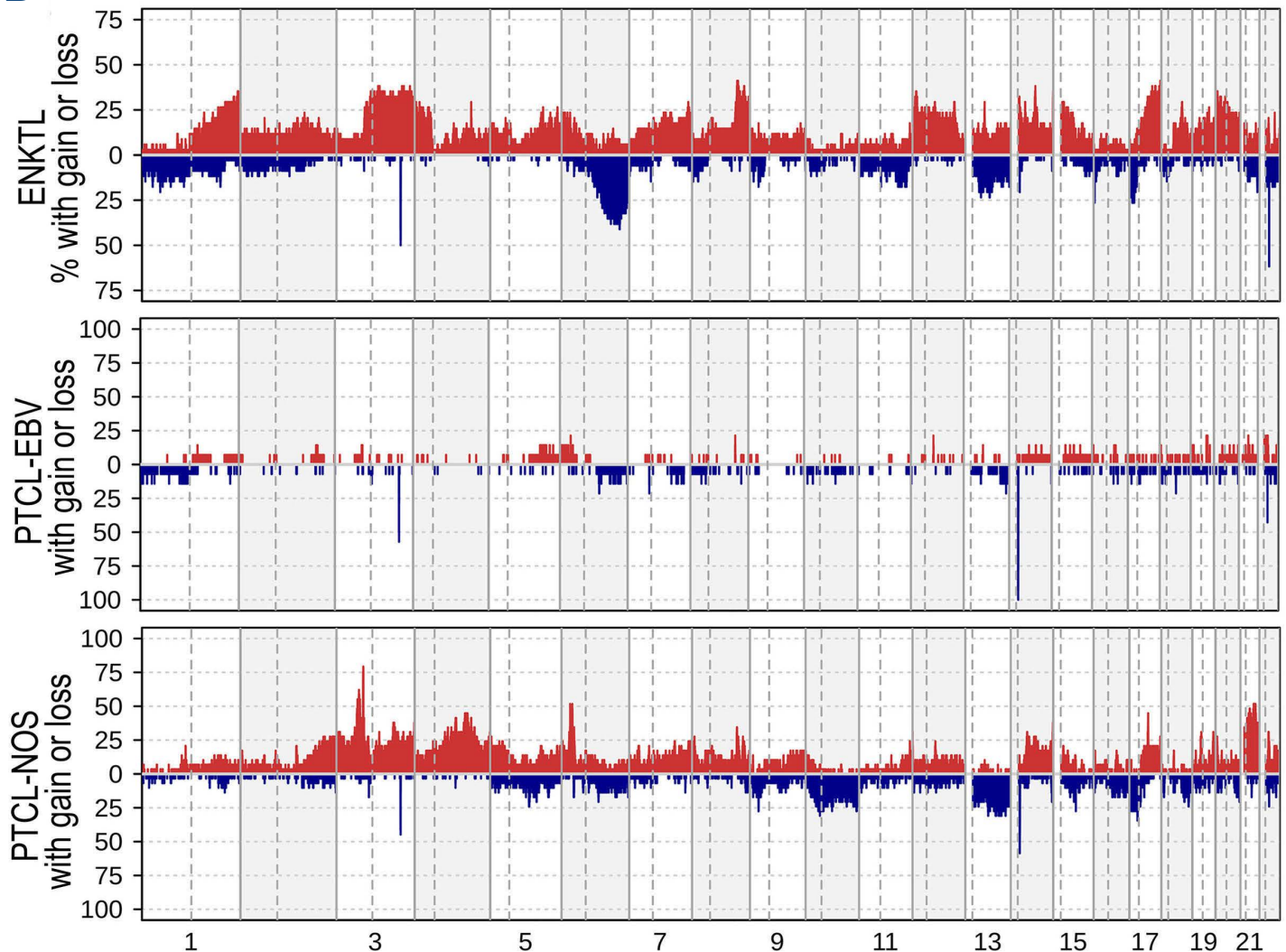

C

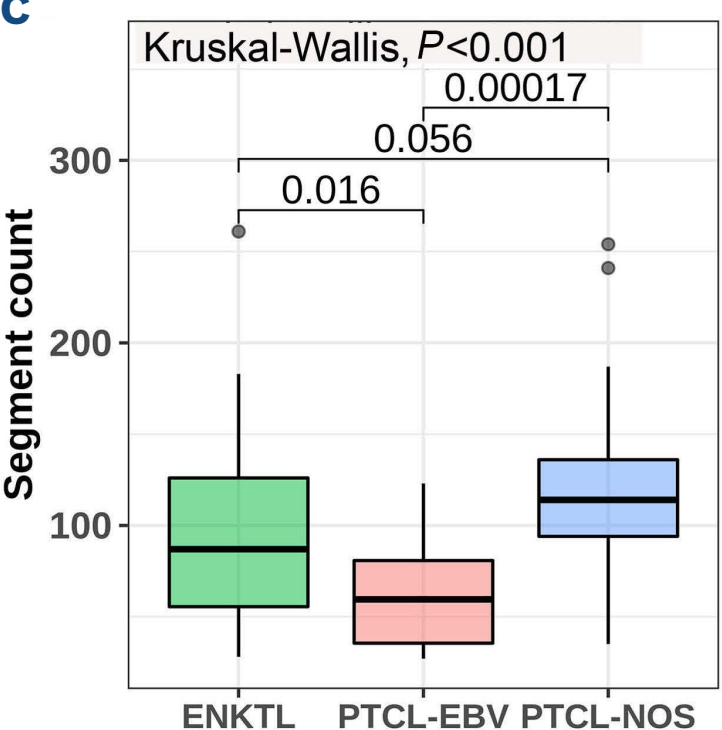

D

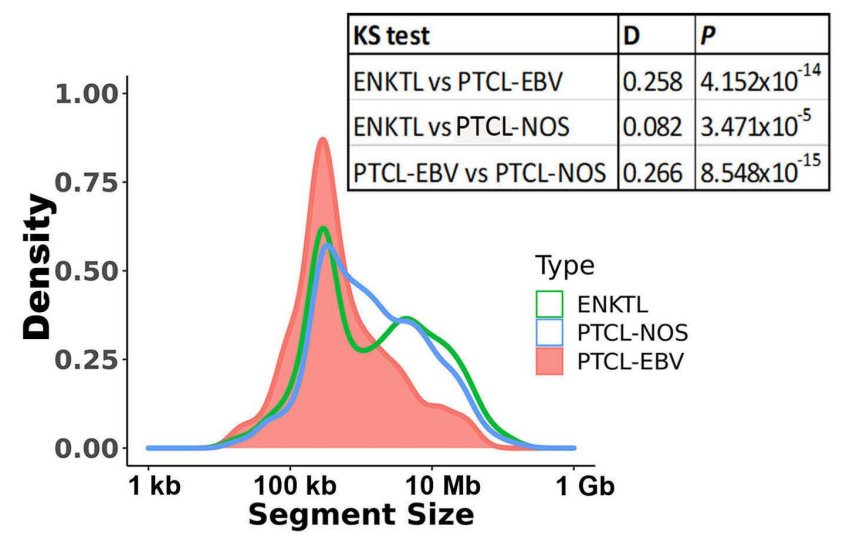

E

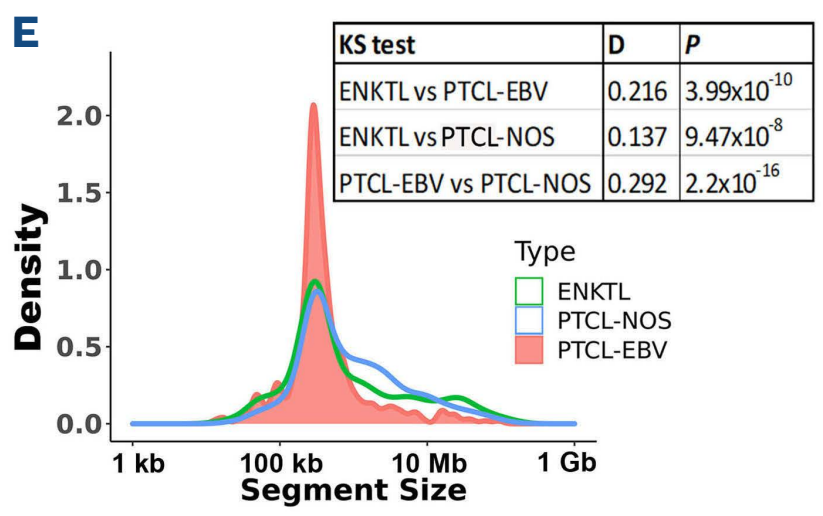

Continued on following page. 
Figure 2. Composite copy number alteration profiles of three disease groups. (A) Composite map showing the focal copy number alteration spectrum in three disease groups. The red and blue represent copy number gain and loss, respectively. Each row represents a genomic locus while each column represents a case. Bars on the right represent the proportion of each disease type in copy number aberrations identified. PTCL-EBV patients had fewer focal copy number aberrations compared to patients with ENKTL and PTCL-NOS. (B) Penetrance plots showing the frequency of gains and losses of genomic loci in ENKTL, PTCL-EBV and PTCL-NOS groups. The X-axis represents chromosome number and the Y-axis indicates the proportion of gain or loss of the corresponding genomic loci within the corresponding population. Red bars denote copy number gains and blue bars denote copy number losses. PTCL-EBV exhibited less frequent genomic alterations compared to other disease groups. (C) Boxplot depicting total copy number segment counts (left), gains only (middle) and losses only (right) across the three diseases. Differences among the three groups were determined using the Kruskal-Wallis test while pairwise comparisons were assessed by the MannWhitney $U$ test ( $P$ values shown). PTCL-EBV displayed lower segment counts compared to ENKTL and PTCL-NOS. (D, E) Copy number segment size distribution of gains (D) and losses (E) in the three disease groups. Statistical significance was determined using a two-sample Kolmogorov-Smirnov test with $\mathrm{P}$ values indicated in the table. PTCL-EBV gain and loss distributions were enriched for smaller copy number segments compared to the other disease groups.

(Figure 2C, right panel). The sizes of gains and losses are known to vary across cancer types and these differences may be attributable to different mutagenic processes. ${ }^{17}$ Pairwise comparisons of copy number aberration size distributions indicated that all three diseases exhibited distinct patterns of gains and losses $(P<0.05$, Kolmogorov-Smirnov test) (Figure 2D, E) with PTCL-EBV showing a propensity to smaller gains ( $300 \mathrm{~kb}$ in size) and a unimodal distribution for losses peaking around $300 \mathrm{~kb}$. To further examine the genomic complexities of the three disease groups, GI and HRD scores were calculated; the former based on the ratio of total length of regions with aberrant copy number and the latter on the loss of heterozygosity, telomere allelic imbalance and large-scale state transitions. ${ }^{11}$ We observed that PTCL-EBV had significantly lower GI and HRD scores compared to ENKTL $(P<0.001$ [Gl], $P=0.004$ [HRD], Mann-Whitney $U)$ and PTCLNOS ( $P=0.0012$ [GI], $P=0.025$ [HRD]) (Figure 3A, B). Despite these lower scores, ploidy levels were similar across all three groups, indicating that these results were not attributable to differential rates of whole genome duplication (Figure $3 \mathrm{C}$ ). The Gl score remained significantly lower in PTCL-EBV T-lineage $(n=13)$ than in ENKTL T-lineage $(n=9)$ cases, indicating that the lower Gl score in PTCL-EBV compared to ENKTL is not related to lineage. Nevertheless, future studies with larger sample sizes will be necessary for a comparison of lineage effects across the disease groups. In line with previous reports describing TP53 alterations co-occurring with increased GI or HRD, 18 we also observed that TP53 losses were associated with higher GI score (Online Supplementary Figure S3) and were less frequent in PTCL-EBV (7.1\%) than in ENKTL (26.5\%; n.s., Fisher exact test) and PTCL-NOS ( 31.4\%; P=0.03) (Online Supplementary Table S5).

Using 14 publicly available Oncoscan datasets (Online Supplementary Table S7), we compared GI and HRD scores of other hematolymphoid neoplasms and solid cancers with those of PTCL-EBV, ENKTL and PTCL-NOS. Oncoscan datasets for T-cell lymphomas were unavailable. GI and HRD scores varied widely across cancer types, with the highest scores in solid cancers and the lowest in chronic myeloid leukemia and pediatric-type follicular lymphoma (Figure 3D). Within hematolymphoid malignancies, high-grade lymphomas, such as Burkitt-like lymphomas, large B-cell lymphomas, ENKTL and PTCL-NOS, had higher GI than low-grade malignancies. Compared to other aggressive Bcell lymphomas, PTCL-EBV demonstrated a remarkably low Gl score (all $P<0.003$ ).

\section{NF-KB and immune pathways are overexpressed in PTCL-EBV}

To explore the biological pathways associated with PTCL$E B V$, we investigated genes that were differentially expressed between PTCL-EBV and ENKTL (EBVVSENKTL) or PTCL-NOS (EBVvsNOS). After filtering non-consensus coding sequence probes, 244 and 95 differentially expressed genes $(P<0.01$, adjusted $P<0.05)$ were identified in EBVVSENKTL and EBVVsNOS, respectively (Online Supplementary Table S8). Interestingly, all 95 EBVvsNOS differentially expressed genes overlapped with the 244 from EBVVsENKTL. As expected, unsupervised hierarchical clustering of these 244 genes revealed three distinct clusters with PTCL-EBV separated from ENKTL and PTCL-NOS (Online Supplementary Figure S4). To assess differentially expressed genes for over-representation of gene ontology terms and protein-protein interactions, genes differentially expressed in EBVVSENKTL and EBVVSNOS were independently submitted to STRING. ${ }^{19}$ Both sets of differentially expressed genes were enriched for numerous immunity-related processes (Online Supplementary Tables S9 and S10) and known protein-protein interactions $(P<0.005)$ (Online Supplementary Figure S5). Subsequent analyses of known gene-gene interactions identified NFKB-associated genes BIRC3, NFKB1, TLR8 and CD27 which are upregulated in PTCL-EBV - as central nodes in the differentially expressed gene networks (Figure 4). To corroborate differential expression data at the protein level, multiplexed immunofluorescence was performed (Figure 5, Online Supplementary Figure S6A) and revealed significantly upregulated expression of BIRC3 and p50 (NFKB1) in tumor (Online Supplementary Figure S6B, C) and non-tumor (Online Supplementary Figure S6E, F) cells of 
A

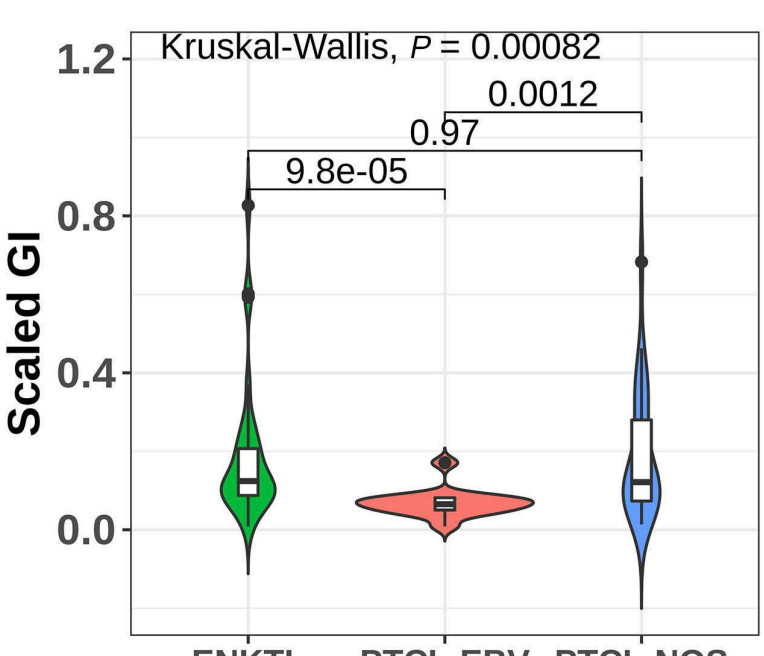

C

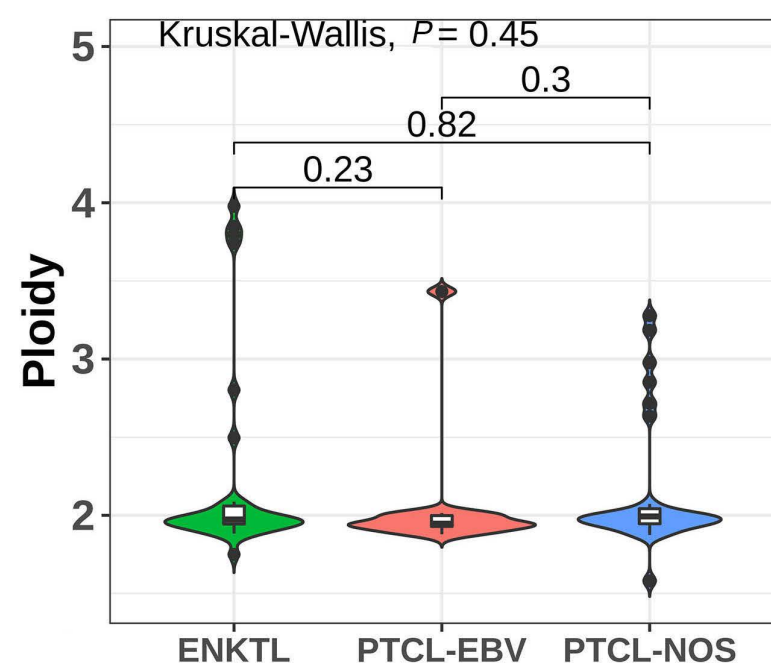

B
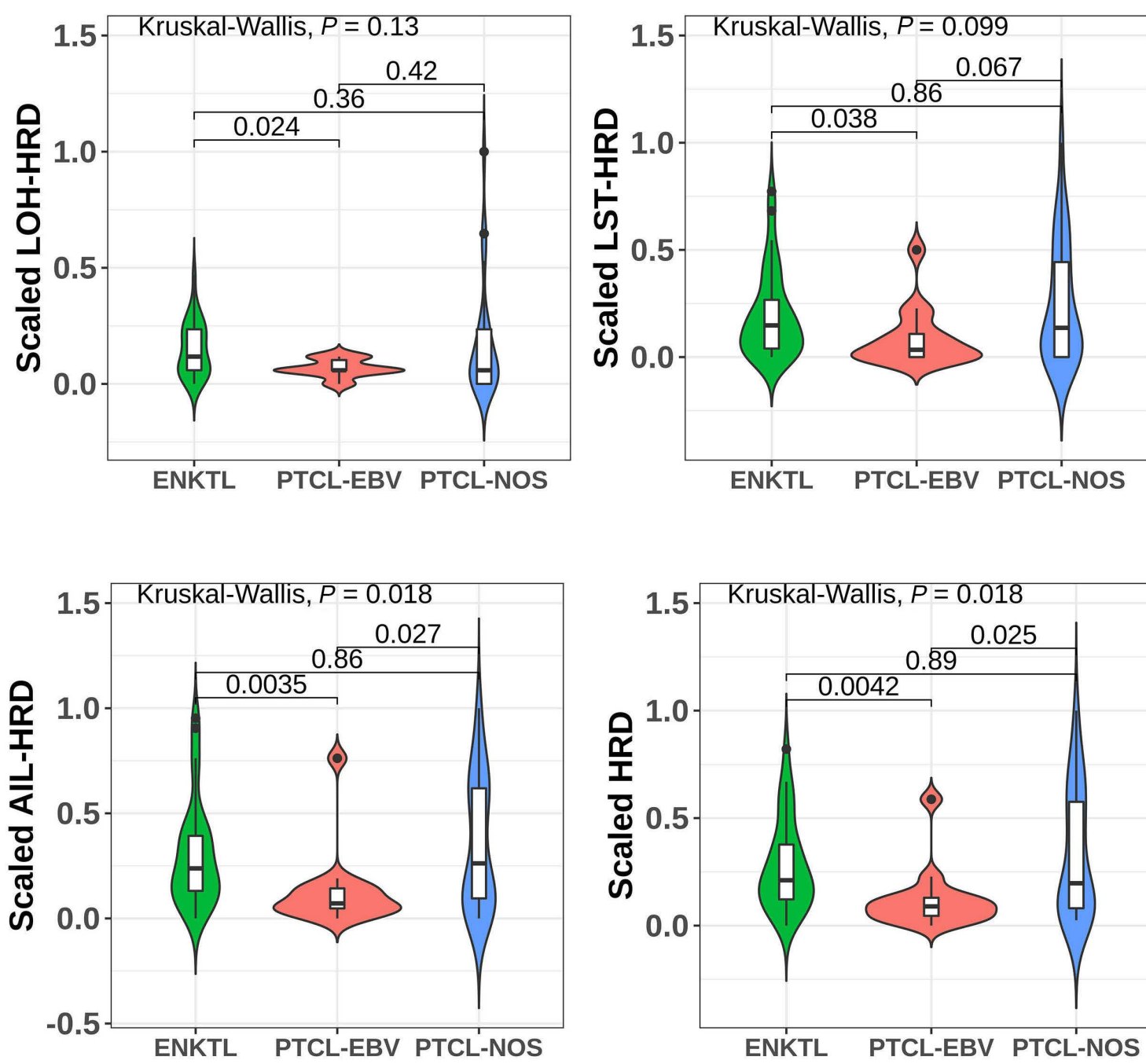

D

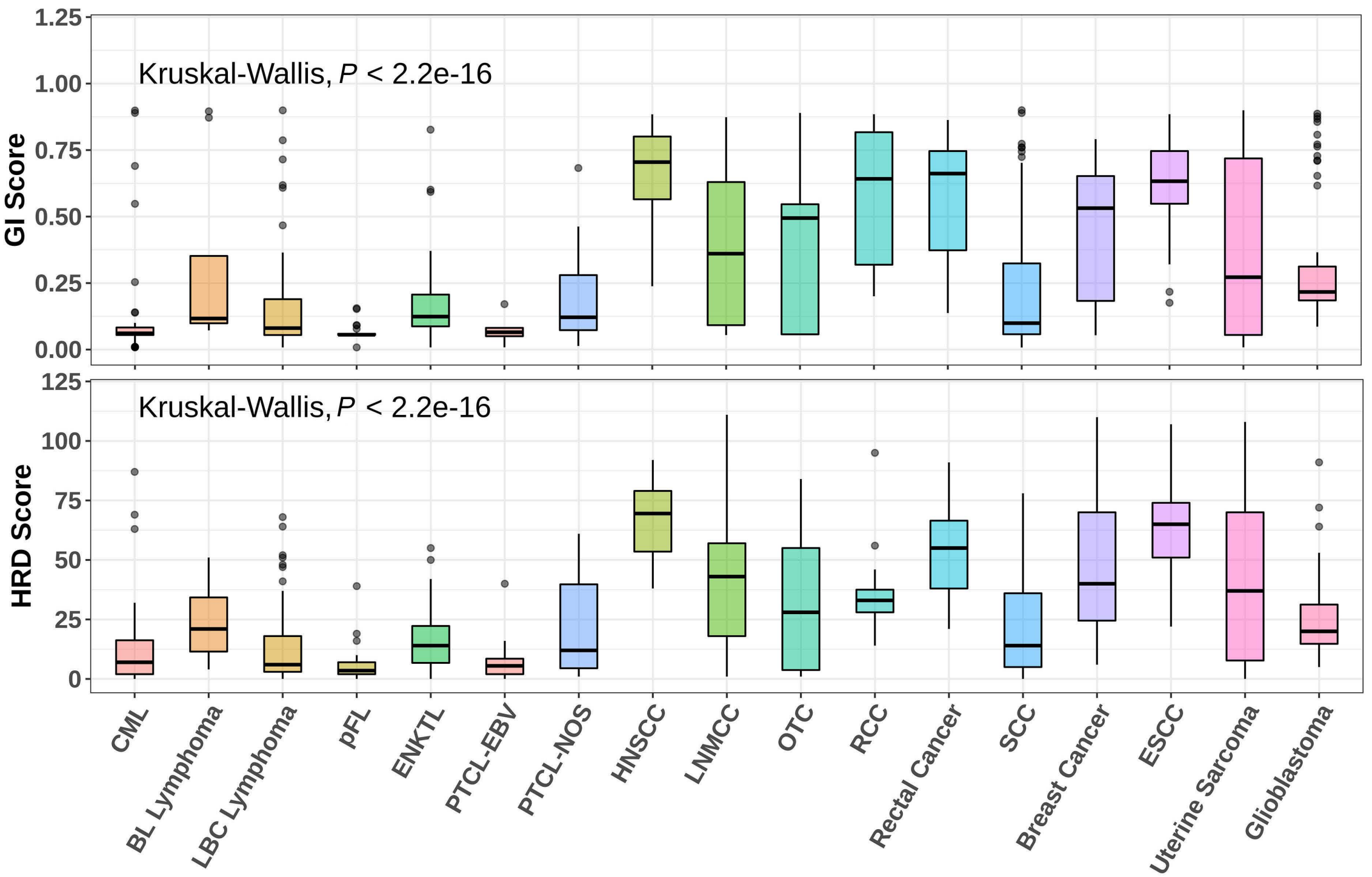

Continued on following page. 
Figure 3. Differences in genomic instability score, homologous recombination deficiency score and ploidy across different disease groups. Segmentation output data from OncoScan microarray ( $n=77$ cases; ENKTL=34, PTCL-EBV=14, PTCL-NOS=29) was analyzed and quantified for scores of (A) GI, (B) LOH-HRD, LST-HRD, AIL-HRD and scaled HRD and (C) ploidy. (D) Comparison of GI- and HRD- scores across different cancer groups also profiled via OncoScan. Oncoscan datasets on T-cell lymphomas were unavailable. Wide variation of GI- and HRD- scores was observed across cancer types with HNSCC having highest scores while CML and pFL had lowest. High-grade lymphomas, such as Burkitt-like lymphomas, large B-cell lymphomas, ENKTL and PTCLNOS had higher GI- and HRD-scores than low-grade lymphomas. Our results showed that PTCL-EBV exhibited significantly lower GI- and HRD- scores among aggressive lymphomas and various solid tumors. Statistical significance was determined using Kruskal-Wallis tests for differences among the three disease groups while Mann-Whitney $U$ tests were used for pairwise comparisons. LOH: loss of heterozygosity; LST: large-scale state transitions; AIL: telomere allelic imbalance; BL: Burkitt-like; CML: chronic myeloid leukemia; ESCC: esophageal squamous cell carcinoma; HNSCC: head and neck squamous cell carcinoma; LBC: large B-cell; LNMCC: lymph node metastases in colon cancer; OTC: oral tongue carcinoma; pFL: pediatric-type follicular lymphoma; RCC: renal cell carcinoma; SCC: synchronous colorectal cancer; HRD: homologous recombination deficiency.

PTCL-EBV compared to ENKTL and PTCL-NOS. CD27 expression was significantly higher in tumor and non-tumor cells (Online Supplementary Figure S6D, G) of PTCL-EBV compared with ENKTL (both $P<0.001$ ) but not with PTCLNOS. Interestingly, the proportions of non-tumor cells which were double positive for CD27/BIRC3, CD27/p50, and triple positive for CD27/p50/BIRC3 within a single nontumor cell, were also significantly higher in PTCL-EBV than in ENKTL and PTCL-NOS (Online Supplementary Figure S6A, H-K).

To further explore NFKB across EBVVSENKTL and EBVvsNOS, we performed gene set enrichment analysis using five curated sets of NFKB target genes (Online Supplementary Methods). We observed consistent and significant upregulation of $\mathrm{NF \kappa B}$ target gene expression in PTCL-EBV compared to ENKTL and PTCL-NOS (Figure 6A). In addition, we found no significant difference in tumor content (Online Supplementary Figure S7A) and observed a rather homogeneous composition of the main immune components of the TME according to computational transcriptome deconvolution across the disease groups (Online Supplementary Figure S7B) using CIBERSORTx. This suggests that the upregulation of immune-related pathways in PTCL-EBV is unlikely to be related to tumor content or TME composition difference between the disease groups. Overall, these results indicate prominent immune pathway upregulation and NFKB activation in PTCL-EBV, suggesting the potential role of persistent NFkB signaling in the disease pathogenesis.

\section{IFN $\gamma$, JAK-STAT and NFKB is upregulation in PTCL-EBV and correlation with PD-L1}

To further elucidate the biological pathways associated with GI, we correlated GI score to the expression of each gene across all samples, which resulted in a list of genes ranked by the Spearman rho. This list was submitted to gene set enrichment analysis to identify hallmark gene sets whose expression was associated with Gl scores (Online Supplementary Table S11). Our top three gene sets interferon_alpha_response (false discovery rate <0.001), interferon_gamma_response (false discovery rate <0.001) and IL6_JAK_STAT3_signaling (false discovery rate $=0.001$ ) (Online Supplementary Figure S8A-C) - displayed inverse correlations, indicating that these immune-related pathways are upregulated in PTCL-EBV and coincide with lower GI scores.

We previously reported that the expression of PD-L1 is higher in PTCL-EBV than in ENKTL. ${ }^{4}$ Given that both interferon_gamma_response (IFN $\gamma$ ) and STAT3 are able to induce PD-L1 expression at both gene and protein levels in cancers, including ENKTL, ${ }^{20,21}$ we correlated the gene expression of IFNY and the IL6_JAK_STAT3 pathway with PDL1 (CD274) to understand mechanisms driving PD-L1 upregulation in our disease groups. We observed a significant correlation between the gene expression of IFN $\gamma$ $(R=0.55, P<0.001)$ and IL6_JAK_STAT3 genes $(R=0.79$, $P<0.001$ ) with CD274 (Figure 6B, C). Similarly, we also assessed the association between NFKB activity and CD274 since NFKB can transcriptionally upregulate $C D 274$ expression. ${ }^{22,23} \mathrm{~A}$ significant correlation between the median expression of NFKB transcriptional target genes and CD274 was also observed ( $R=0.69, P<0.001)$ (Figure 6D). Overall, it is possible that the upregulation of PD-L1 in PTCL-EBV may be related to activation of IFN $\gamma$, IL6_JAK_STAT3 and NFKB.

\section{EBV miRNA are downregulated in PTCL-EBV compared to ENTKL}

Since ENKTL and PTCL-EBV are both associated with EBV, we compared EBER expression, tumor content (via Oncoscan), and EBV miRNA (via qPCR) in both diseases. There was no significant difference in EBER positivity (Online Supplementary Figure S9A) or tumor content (Online Supplementary Figure $S 9 B$ ) between the two diseases. Based on the gene expression of EBNA1, EBNA2, LMP1 and LMP2A by RT-PCR, the majority (9/13, 69\%) of PTCL-EBV showed a type 2 EBV latency pattern, while four cases (31\%) demonstrated a type 3 latency pattern (Online Supplementary Table S12). Interestingly, PTCL-EBV $(n=9)$ displayed a widespread lower EBV miRNA expression compared to ENKTL $(n=15)$ and clustered separately from it (Online Supplementary Figure S10). The expression of 32 of 42 (76\%) EBV 
miRNA was significantly lower in PTCL-EBV (adjusted $P<0.05$, t-test) (Online Supplementary Table S13).

To better understand the potential transcriptional impact of this differential miRNA regulation, we correlated the expression of each differentially expressed EBV miRNA and its predicted targets. Given that miRNA negatively regulate target $m R N A,{ }^{24}$ all target genes that were negatively correlated (adjusted $P<0.05$ ) to their corresponding EBV miRNA were analyzed $(n=172)$ (Online Supplementary Table S14). Strikingly, the pathways most enriched (adjusted $P<0.05$ ) within this set of genes represented either immunity or interferon signaling (Online Supplementary Table S15). After calculating a gene expression index (median expression) for these target genes, we observed significantly higher expression in PTCL-EBV ( $P=0.03$, Mann-Whitney U) (Online Supplementary Figure S11), which is consistent with lower EBV miRNA expression in this group. Overall, these results suggest that downregulation of EBV miRNA is unlikely to be related to a difference in tumor or EBER content and

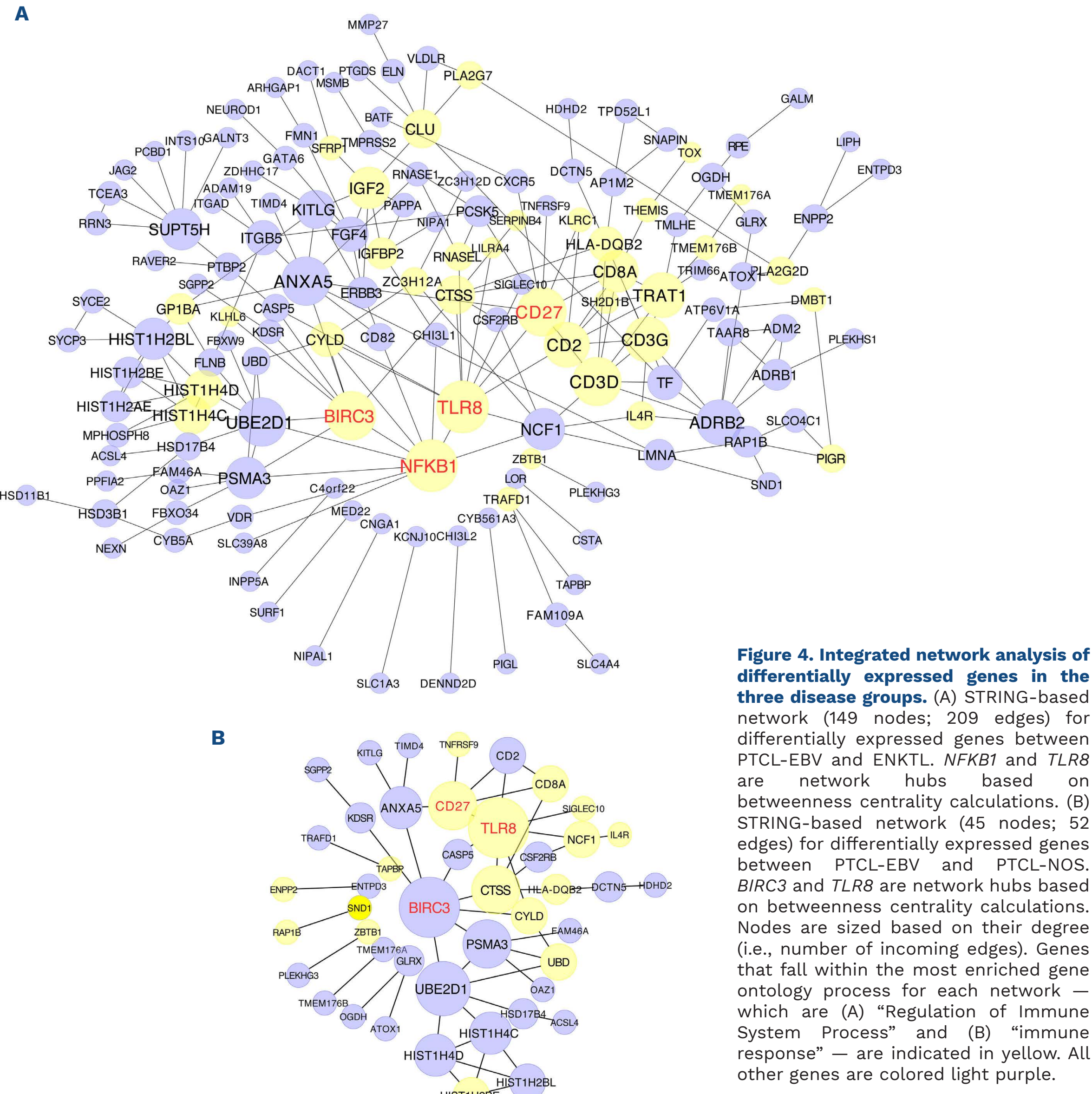




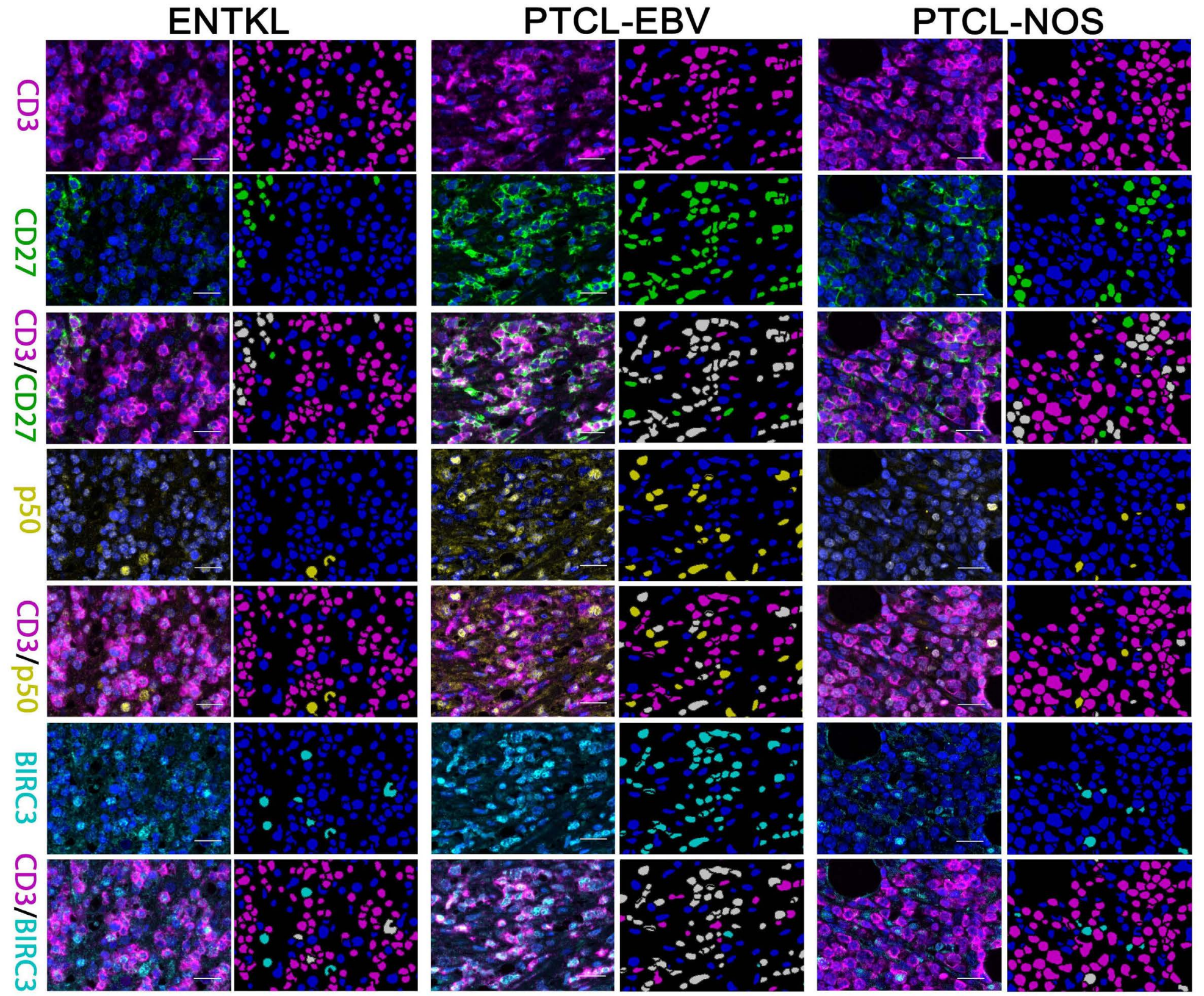

Figure 5. Multiplex immunofluorescence analysis of BIRC3, p50 (NFKB1) and CD27 across all three diseases. (A) Protein expression of CD27, p50 (NFKB1) and BIRC3 in ENKTL (left panel), PTCL-EBV (middle panel) and PTCL-NOS (right panel) using multiplexed immunofluorescence. For each panel, the left column represents the multiplexed immunofluorescence staining and the right column shows the corresponding multispectral analysis masks. PTCL-EBV showed higher expression of CD27 (membrane, green), p50 (NFKB1) (nuclear, yellow) and BIRC3 (nuclear, cyan), compared to ENKTL and PTCL-NOS. CD27 ${ }^{+} \mathrm{CD} 3^{+}$cells are white while $C D 27^{+} C D 3^{-}$are green in CD3/CD27 masks. $P 50^{+} C D 3^{+}$cells are white while $P 50^{+}$CD $3^{-}$are yellow in $C D 3 / p 50$ masks. $B I R C 3^{+} C D 3^{+}$cells are white while BIRC $3^{+} C D 3^{-}$cells are cyan in $\mathrm{CD} 3 / \mathrm{BIRC} 3$ masks. The scale bars indicate $100 \mu \mathrm{m}$.

could, in part, contribute to the distinctive pattern of immune gene transcription in PTCL-EBV.

\section{PTCL-EBV showed frequent mutations of TET2, PIK3CD and STAT3}

Based on the evidence that Gl, gene expression, as well as EBV-miRNA patterns can delineate a distinct profile for PTCL-EBV, we further investigated whether this disease harbors mutations in known driver genes of PTCL-NOS, ENKTL and solid cancers using a 35-gene T/NK lymphoid panel and 484-gene NovoPM ${ }^{\top M} 2.0$ assay (total 500 genes with 19 common genes covered in both panels).

The most commonly mutated gene was TET2 (9/14, 64\%) followed by PIK3CD (3/9, 33\%), STAT3 (3/16, 19\%), DDX3X $(2 / 10,20 \%)$ and PTPRD $(2 / 11,18 \%)$ (Online Supplementary Figure S12). The variant allele frequency ranges for TET2 in the NovoPM ${ }^{\mathrm{TM}} 2.0$ panel and T/NK lymphoid panel were $22.2 \%-40.7 \%$ and $22 \%-76 \%$, respectively. Given the tumor purity of our samples and the high frequency of TET2 mutations and their associated variant allele frequencies, it is unlikely that these mutations are attributable to clonal hematopoiesis of indeterminate potential (CHIP). ${ }^{25} \mathrm{How}$ ever, in the absence of blood samples from these patients, we cannot entirely rule out CHIP as a possible source of TET2 mutation in some of our PTCL-EBV cases. Eight out of nine cases positive for TET2 mutation were positive for CD8, indicating that they were not PTCL with T-follicular helper phenotype. Interestingly, TP53 mutations, commonly present in ENKTL, were not detected in our PTCLEBV cases. The median number of mutations detected was 2.5 per sample (range, 1 to 11 ).

The tumor mutational burden score ranges from 0 to 7.86 mutations/MB (median 4.285 mutations/MB) (Online Supplementary Figure S13A). A tumor mutational burden of less than 5 is regarded as a low mutational burden in some studies. ${ }^{26,27}$ Eleven samples tested had a microsatel- 
A

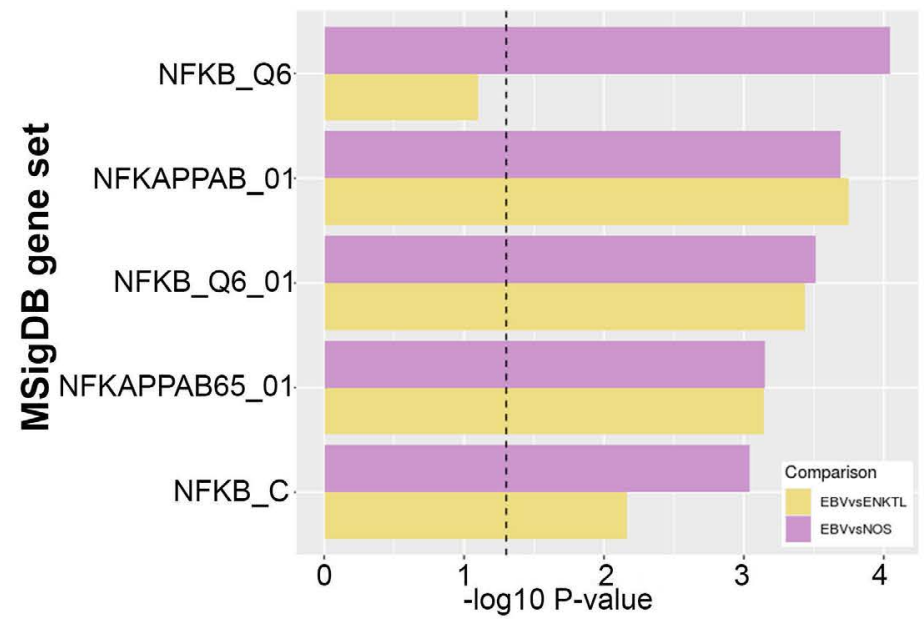

C

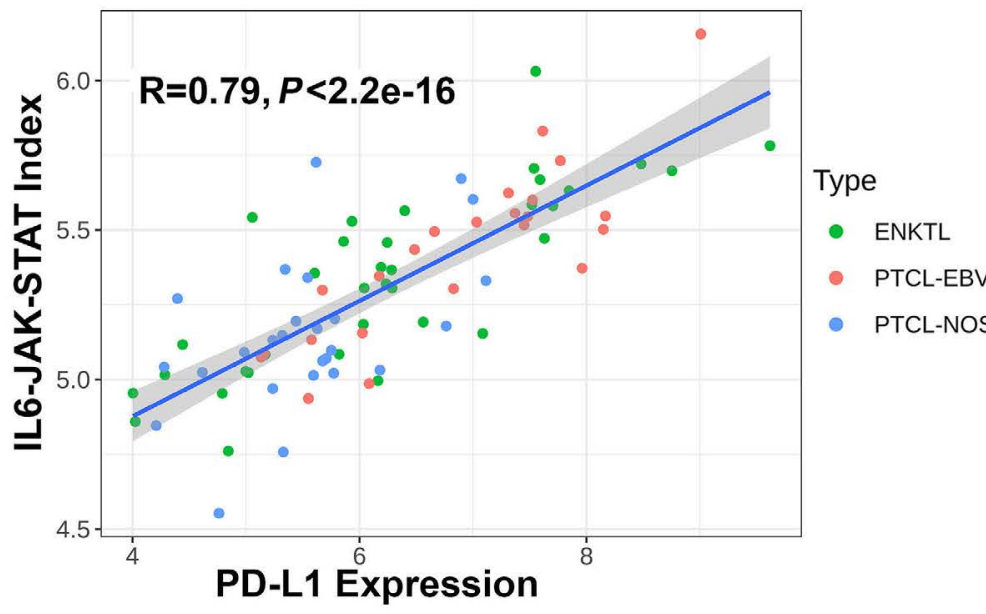

B
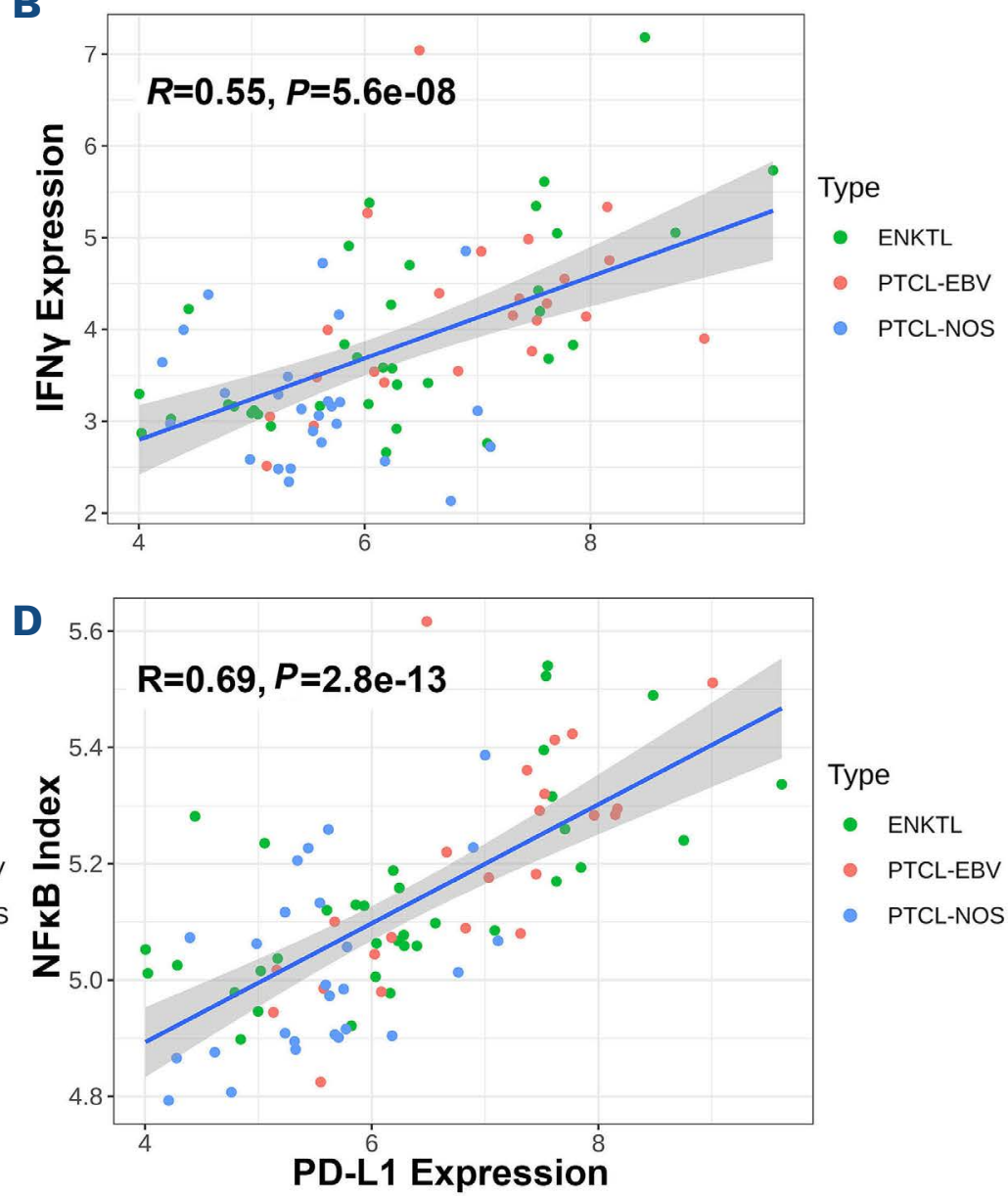

$\mathbf{E}$

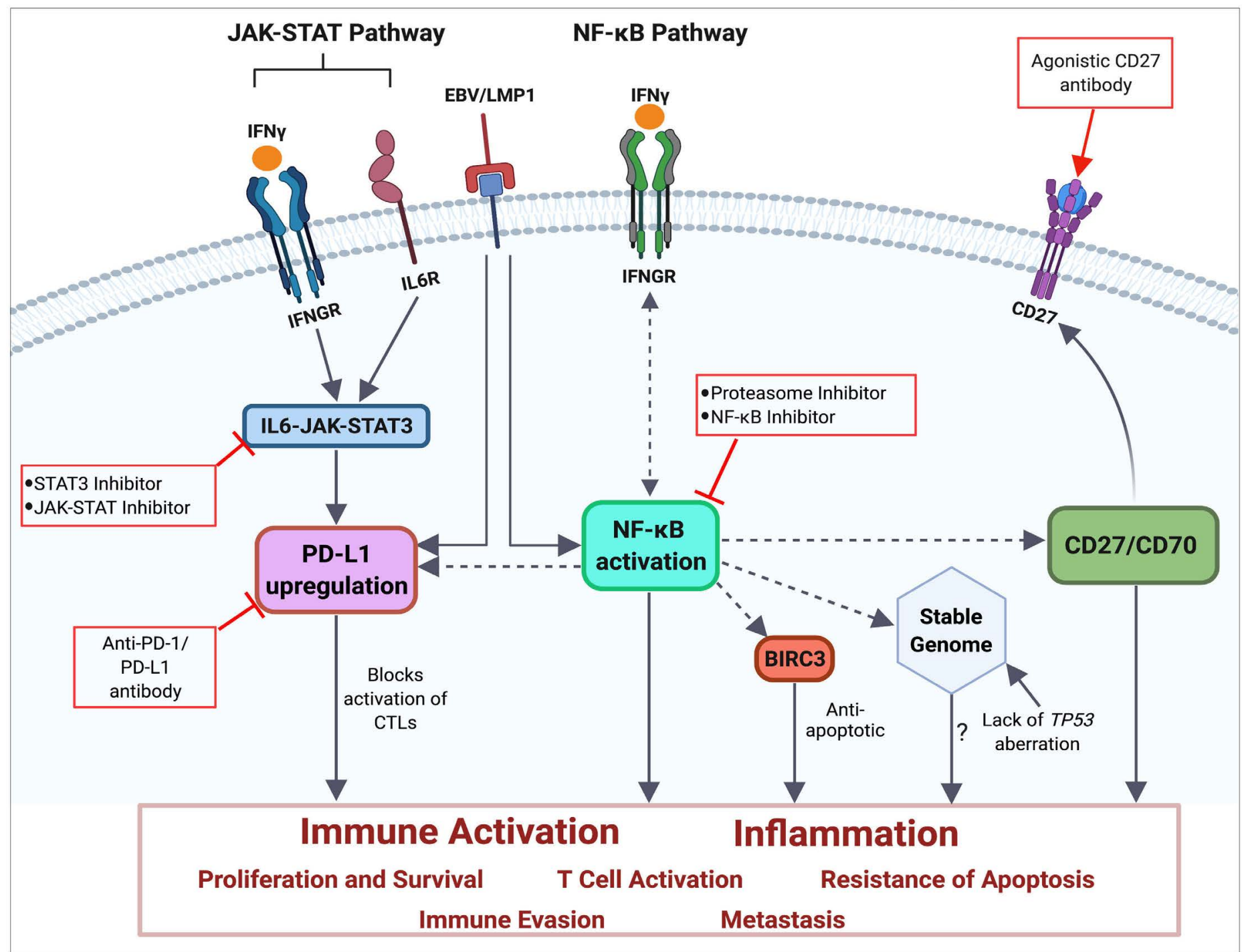

Figure 6. PTCL-EBV demonstrates NFKB transcriptional target gene upregulation and PD-L1 (CD274) expression associates with immune pathway activation across all three diseases. (A) Gene set enrichment analysis (GSEA) comparing PTCL-EBV to ENKTL (EBVVSENKTL) and PTCL-NOS (EBVvsNOS) across five sets of NFKB transcriptional target genes. Genes were ranked by their relative expression differences in EBVVSENKTL and EBVvSNOS then submitted to GSEA. All enrichment scores were positive indicating target gene upregulation in PTCL-EBV compared to ENKTL and PTCL-NOS. The vertical dashed line represents a 0.05 $P$ value threshold. Correlation of PD-L1 (CD274) expression with (B) IFN $\gamma$, (C) the IL6_JAK_STAT pathway and (D) NFKB target gene expression across all three diseases. Our results showed that expression of IFN $\gamma$ and IL6_JAK_STAT genes (median) correlated with PD-L1 gene expression. Taking the union of the five aforementioned gene sets, there was also a positive correlation between 
NFKB transcriptional target gene expression (median) and PD-L1 expression. Correlations were assessed using the Spearman method. Rho and $P$ values are shown. (E) Possible model of PTCL-EBV pathogenesis involving the activation of the NFKB pathway and upregulation of PD-L1, BIRC3, and CD27. BIRC3 plays key roles in the regulation of NFKB signaling and apoptosis. CD27 contributes to anti-tumor cytotoxic T-cell lymphocyte response in the host, T-cell exhaustion, compromise in antitumor immunity. In addition, EBV LMP1 and upregulation of IFN $\gamma$ and IL6_JAK_STAT3 could also contribute to PDL1 overexpression in PTCL-EBV. Activation of these signaling pathways eventually contributes to inflammation, T-cell and immune activation, thereby promoting proliferation and survival, metastasis, immune evasion and oncogenesis. Some of these genes and signaling pathways may serve as potential therapeutic targets for PTCL-EBV and are indicated in red boxes. Dotted lines indicate hypothetical postulations which have not been experimentally validated in PTCL-EBV. Figure created with BioRender.com.

lite instability score below the threshold score of 0.4 (median 0.1882) and are regarded as being microsatellite stable (Online Supplementary Figure S13B).

\section{PTCL-EBV is more aggressive and shows lower genomic instability than cytotoxic PTCL-NOS}

Given that PTCL-EBV is characterized by a cytotoxic phenotype, ${ }^{2,5}$ we attempted to determine whether PTCL-EBV shows similarities with cytotoxic PTCL-NOS. We performed multivariate survival analysis and compared the GI and HRD scores, between PTCL-EBV, PTCL-NOS cytotoxic $(n=15)$ and non-cytotoxic $(n=11)$ cases. Interestingly, we observed that PTCL-EBV was significantly more aggressive than cytotoxic PTCL-NOS (HR=0.22, 95\% Cl: 0.08-0.58, $P=0.002$ ) but not non-cytotoxic PTCL-NOS (Online Supplementary Table S16).

PTCL-EBV exhibited significantly lower Gl scores compared to both cytotoxic $(P=0.041)$ and non-cytotoxic PTCLNOS $(P<0.001)$ (Online Supplementary Figure S14). HRD scores were lower in PTCL-EBV than in non-cytotoxic PTCL-NOS $(P=0.021)$ but not cytotoxic PTCL-NOS. No significant difference was detected in survival, ploidy, and GI and HRD scores between cytotoxic and non-cytotoxic PTCL-NOS and there were no genes significantly differently expressed between the two. Overall, our results show a significant difference in overall survival and GI score between PTCL-EBV and cytotoxic PTCL-NOS, suggesting biological differences between these two tumors.

\section{Discussion}

PTCL-EBV is a rare and aggressive tumor that occurs mostly in East Asia. It is a poorly understood lymphoma that is not recognized yet as a distinct entity but is considered as a variant of PTCL-NOS in the current WHO classification. ${ }^{5}$ In line with published literature, all cases of PTCL-EBV in this study are from South-East/East Asia. Herein, we performed an integrative analysis to compare PTCL-EBV with ENKTL and PTCL-NOS, and our findings demonstrated distinctive features in PTCL-EBV which set it apart from the other two tumors. Our results support the consideration of PTCL-EBV as a distinct entity in the WHO classification.

Copy-number analysis of PTCL-EBV demonstrated a strik- ing profile characterized by not only significantly fewer copy number aberrations, but also a different size distribution compared to ENKTL and PTCL-NOS. Remarkably, PTCL-EBV was more genomically stable than the other two diseases, as reflected by lower GI and HRD scores. High-grade lymphomas had higher GI and HRD scores than low-grade malignancies, compatible with reports documenting that $\mathrm{Gl}$ is associated with aggressive lymphomas and high-grade transformation. ${ }^{28,29}$ Similarly, within and across a variety of solid tumors, increased copy numberbased Gl is associated with poor outcomes. ${ }^{30}$ In this regard, the uniformly low GI and HRD scores in PTCL-EBV belies its aggressive behavior.

The discrepancy between low GI and aggressive behavior is novel but difficult to explain. Given the prominent immune-related gene expression profile, it is reasonable to hypothesize that the aggressiveness of PTCL-EBV may be related to a cancer-promoting inflammation which blocks anti-tumor immunity and directs the tumor microenvironment toward a tumor-permissive state. ${ }^{31}$ Interestingly, our findings showed that PTCL-EBV has few TP53 mutations and has a paucity of TP53 losses compared to ENKTL and PTCL-NOS. Given the paucity of TP53 alterations in PTCLEBV, it is possible that low GI in PTCL-EBV may be related to an unperturbed p53 tumor suppressor function, which is necessary to preserve genomic stability and integrity through cell-cycle arrest, senescence and apoptosis. ${ }^{32}$ Given that chromosome instability in diffuse large B-cell lymphomas can be suppressed by NFKB activation, ${ }^{33}$ it is conceivable that NFKB activation in PTCL-EBV may also contribute towards the low GI but high aggressiveness of this tumor. ${ }^{34,35}$ Alternatively, there may be epigenetic deregulation driving oncogenesis in PTCL-EBV, which was not investigated in this study. ${ }^{36}$

It is now known that cancer cells interact with surrounding stromal and immune cells to form a pro-tumorigenic inflammatory tumor microenvironment. ${ }^{31}$ Compared to ENKTL and PTCL-NOS, PTCL-EBV is characterized by striking activation of immune-related pathways, in particular, upregulation of NFKB and its associated genes, BIRC3, NFKB1 and CD27, in both tumor and non-tumor cells. The upregulation of these three markers, either singly or colocalized with each other, suggests that there are different subsets of immune cells with varied combinations of BIRC3, p50 and CD27 expression in PTCL-EBV, and that the 
$N F-\kappa B$ signaling pathway likely plays a role in promoting an active inflammatory tumor microenvironment in PTCLEBV. NFKB plays a key role in linking inflammation to cancer ${ }^{31}$ and targets different immune cells to modulate inflammation, tumorigenesis and metastasis. ${ }^{37}$ The pronounced immune activation and $\mathrm{NF} \kappa \mathrm{B}$ upregulation in PTCL-EBV may augment the local inflammatory state and suppress cytotoxic T-lymphocyte effector function, resulting in an immunosuppressive tumor microenvironment that enhances immune evasion and aggressive behavior. ${ }^{38,39}$ While CD27 normally activates NFKB, promotes cell survival and enhances $\mathrm{T}$ - and $\mathrm{B}$-cell receptor-mediated proliferative signals, dysregulation of CD27 signaling can result in T-cell exhaustion and dysfunction. ${ }^{40,41}$ Interestingly, we found that EBV-miRNA were predominantly downregulated in PTCL-EBV compared to ENKTL, which may further contribute to the inflammatory state with overexpression of immune-related genes. ${ }^{42}$ Based on our correlative data, we propose a model of PTCL-EBV pathogenesis involving NFKB activation and upregulation of PDL1, BIRC3 and CD27 (Figure 6E). Some of these pathways may serve as potential therapeutic targets for PTCL-EBV as drugs targeting JAK-STAT, NFKB, STAT3, IFN $\gamma$, PD1/PD-L1 are either approved by FDA for different cancers or are being evaluated in clinical trials for lymphomas. ${ }^{43-45}$

The upregulation of the immune checkpoint protein PDL1 in PTCL-EBV, inducible by IFN $\gamma$, may block the activation of the cytotoxic T-cell lymphocyte antitumor response, lead to T-cell exhaustion and promote immune evasion. ${ }^{23,46}$ In contrast to other lymphomas, this upregulation is unrelated to 9 q24.1 gain. ${ }^{47}$ Interestingly, we identified a significant correlation between the gene expression of PD-L1 and its known transcriptional-regulators: IFN $\gamma$, IL6_JAK_STAT3 and NFKB, The overexpression of PD-L1 in PTCL-EBV, possibly a result of the upregulation of IFN $\gamma$, IL6_JAK_STAT3 and NFKB pathways, ${ }^{20,23,37}$ suggests that targeting the PD-1/PD-L1 axis $^{43}$ may be a potentially effective therapeutic approach. Nevertheless, understanding the mechanisms causing PD-L1 upregulation, including structural variations in the 3' untranslated region of $P D L 1,{ }^{48}$ is essential for the design of more effective treatment strategies. $^{23}$

Our data demonstrate that the majority of PTCL-EBV cases show a type 2 EBV latency program, ${ }^{15}$ similar to that of ENKTL and other EBV-associated T/NK lymphoproliferative diseases. ${ }^{49,50}$ A minority revealed a type 3 latency pattern. It remains unclear whether this may reflect underlying immunosuppression as it is known that, in a subset of patients, PTCL-EBV is associated with autoimmune conditions, viral infections or diabetes mellitus which may impair the host's immune responses., ${ }^{1,51}$ The downregulation of EBV miRNA in PTCL-EBV compared to ENKTL suggests a difference in the EBV biology between the two diseases, although factors contributing to downregulation of EBV miRNA have yet to be elucidated. Given that the predicted targets of the differentially expressed EBV miRNA are significantly enriched for immune-related pathways, it is tempting to postulate that the lower expression of EBV miRNA may contribute to the persistent expression of the many immune pathways in PTCL-EBV compared to ENKTL since miRNA are known to negatively regulate transcriptional gene expression. ${ }^{24}$ Nevertheless, the complex interplay between EBV miRNA, viral and cellular target genes in EBV-associated T/NK-cell lymphomas requires further investigation.

In summary, PTCL-EBV is an aggressive lymphoma characterized by minimal Gl, immune-related gene expression as well as activation of NFKB and its associated genes. While further studies are needed to corroborate our proposed model, these findings highlight the importance of the crosstalk between tumor and microenvironment, provide new insights hinting at the disease pathogenesis and offer potential new therapeutic targets for this aggressive disease.

\section{Disclosures}

No conflicts of interest to disclose.

\section{Contributions}

CMMW and SC performed research, analyzed data and wrote the manuscript. TP, SML, WZ, LCYL, ADJ, W-JC and $C T$ contributed to data interpretation and analysis. S-NC, $C-K L$ and $S F$ performed histological experiments. T-HC, $K H K B, S G, S L, F Z$ and $F I$ contributed to bioinformatics analysis. Y-HH, SK, SN, ET, Y-HK, JDK, S-SC, RKHA-Y, S-YT, S-TL, LMP, SDM and CKO selected cases and acquired data. LQM and FO performed the mutational analysis. JJP analyzed and interpreted data and wrote the manuscript. S$B N$ designed the project, analyzed data, and wrote and finalized the manuscript.

\section{Acknowledgments}

SBN is supported by the National Medical Research Council, Clinician Scientist Award, (CSAINV17nov016, WBS R-179-000-063-213), National Medical Research Council Open Fund Large Collaborative Grant, Singapore IYMPHoma translational study (SYMPHONY) (NMRC OFLCG18May-0028), and NUSMed Post-Doctoral Fellowship (PDF) (NUHSRO/2019/036/PDF/09). JJP is supported by the National Research Foundation Singapore and the Singapore Ministry of Education under its Research Centers of Excellence initiative. The computational work for this article was partially performed on resources of the National Supercomputing Center, Singapore (https://www.nscc.sg).

\section{Data-sharing statement}

De-identified data used in the preparation of this manuscript are available upon request. 


\section{References}

1. Ko YH, Chan JKC, Quintanilla-Martinez L. Virally associated Tcell and NK-cell neoplasms. In: Jaffe ES, Arber DA, Campo E, Harris NL, Quintanilla-Martinez L, editors. Haematopathology. Elsevier, Philadelphia 2017;565-598.

2. Kato S, Asano N, Miyata-Takata T, et al. T-cell receptor (TCR) phenotype of nodal Epstein-Barr virus (EBV)-positive cytotoxic T-cell lymphoma (CTL): a clinicopathologic study of 39 cases. Am J Surg Pathol. 2015;39(4):462-471.

3. Kato S, Takahashi E, Asano N, et al. Nodal cytotoxic molecule (CM)-positive Epstein-Barr virus (EBV)-associated peripheral T cell lymphoma (PTCL): a clinicopathological study of 26 cases. Histopathology. 2012;61(2):186-199.

4. Ng SB, Chung TH, Kato S, et al. Epstein-Barr virus-associated primary nodal T/NK-cell lymphoma shows a distinct molecular signature and copy number changes. Haematologica. 2018;103(2):278-287.

5. Pileri SA, Weisenburger DD, Sng I, et al. Peripheral T-cell lymphoma, NOS. In: Swerdlow SH, Campo E, Harris NL, et al., eds. WHO Classification of Tumours of Haematopoietic and Lymphoid Tissues. International Agency for Research on Cancer; Lyon. 2017:403-407.

6. Pikor L, Thu K, Vucic E, Lam W. The detection and implication of genome instability in cancer. Cancer Metastasis Rev. 2013;32(3-4):341-352.

7. Ahmad SS, Ahmed K, Venkitaraman AR. Science in focus: genomic instability and its implications for clinical cancer care. Clin Oncol. 2018;30(12):751-755.

8. Oon ML, Lim JQ, Lee B, et al. T-cell lymphoma clonality by copy number variation analysis of T-cell receptor genes. Cancers (Basel). 2021;13(2):340.

9. Lee CS, Bhaduri A, Mah A, et al. Recurrent point mutations in the kinetochore gene KNSTRN in cutaneous squamous cell carcinoma. Nat Genet. 2014;46(10):1060-1062.

10. Mermel CH, Schumacher SE, Hill B, Meyerson ML, Beroukhim R, Getz G. GISTIC2.0 facilitates sensitive and confident localization of the targets of focal somatic copy-number alteration in human cancers. Genome Biol. 2011;12(4):R41.

11. Sinha S, Mitchell KA, Zingone A, et al. Higher prevalence of homologous recombination deficiency in tumors from African Americans versus European Americans. Nat Cancer. 2020;1(1):112-121.

12. Cheadle C, Vawter MP, Freed WJ, Becker KG. Analysis of microarray data using $Z$ score transformation. J Mol Diagn. 2003;5(2):73-81.

13. Kato S, Yamashita D, Nakamura S. Nodal EBV+ cytotoxic T-cell lymphoma: a literature review based on the 2017 WHO classification. J Clin Exp Hematop. 2020;60(2):30-36.

14. Ha SY, Sung J, Ju H, et al. Epstein-Barr virus-positive nodal peripheral T cell lymphomas: clinicopathologic and gene expression profiling study. Pathol Res Pract. 2013;209(7):448-454.

15. Takahashi E, Asano N, Li C, et al. Nodal T/NK-cell lymphoma of nasal type: a clinicopathological study of six cases. Histopathology. 2008;52(5):585-596.

16. Gong Q, Wang C, Zhang W, et al. Assessment of T-cell receptor repertoire and clonal expansion in peripheral T-cell lymphoma using RNA-seq data. Sci Rep. 2017;7(1):11301.

17. Li Y, Roberts ND, Wala JA, et al. Patterns of somatic structural variation in human cancer genomes. Nature. 2020;578(7793):112-121.

18. Pitt JJ, Riester M, Zheng Y, et al. Characterization of Nigerian breast cancer reveals prevalent homologous recombination deficiency and aggressive molecular features. Nat Commun. 2018;9(1):4181.

19. Szklarczyk D, Gable AL, Lyon D, et al. STRING v11: proteinprotein association networks with increased coverage, supporting functional discovery in genome-wide experimental datasets. Nucleic Acids Res. 2019;47(D1):D607-D613.

20. Song TL, Nairismägi M-L, Laurensia $Y$, et al. Oncogenic activation of the STAT3 pathway drives PD-L1 expression in natural killer/Tcell lymphoma. Blood. 2018;132(11):1146-1158.

21. Garcia-Diaz A, Shin DS, Moreno BH, et al. Interferon receptor signaling pathways regulating PD-L1 and PD-L2 expression. Cell Rep. 2017;19(6):1189-1201.

22. Asgarova A, Asgarov K, Godet Y, et al. PD-L1 expression is regulated by both DNA methylation and NF-kB during EMT signaling in non-small cell lung carcinoma. Oncoimmunology. 2018;7(5):e1423170.

23. Shklovskaya E, Rizos H. Spatial and temporal changes in PD-L1 expression in cancer: the role of genetic drivers, tumor microenvironment and resistance to therapy. Int $\mathrm{J}$ Mol Sci. 2020;21(19):7139

24. Ebert MS, Sharp PA. Roles for microRNAs in conferring robustness to biological processes. Cell. 2012;149(3):515-524.

25. Ferrone CK, Blydt-Hansen M, Rauh MJ. Age-associated TET2 mutations: common drivers of myeloid dysfunction, cancer and cardiovascular disease. Int J Mol Sci. 2020;21(2):626

26. Riviere P, Goodman AM, Okamura R, et al. High tumor mutational burden correlates with longer survival in immunotherapy-naïve patients with diverse cancers. Mol Cancer Ther. 2020;19(10):2139-2145.

27. Liang WS, Vergilio J-A, Salhia B, et al. Comprehensive genomic profiling of Hodgkin lymphoma reveals recurrently mutated genes and Increased mutation burden. Oncologist. 2019;24(2):219-228.

28. Kamranvar SA, Gruhne B, Szeles A, Masucci MG. Epstein-Barr virus promotes genomic instability in Burkitt's lymphoma. Oncogene. 2007;26(35):5115-5123.

29. Nagy M, Balázs M, Adám Z, et al. Genetic instability is associated with histological transformation of follicle center lymphoma. Leukemia. 2000;14(12):2142-2148.

30. Hieronymus $\mathrm{H}$, Murali R, Tin A, et al. Tumor copy number alteration burden is a pan-cancer prognostic factor associated with recurrence and death. Elife. 2018;7:e37294.

31. Taniguchi $\mathrm{K}$, Karin M. NF-KB, inflammation, immunity and cancer: coming of age. Nat Rev Immunol. 2018;18(5):309-324.

32. Yeo CQX, Alexander I, Lin Z, et al. p53 maintains genomic stability by preventing interference between transcription and replication. Cell Rep. 2016;15(1):132-146.

33. Ramachandiran S, Adon A, Guo X, et al. Chromosome instability in diffuse large $B$ cell lymphomas is suppressed by activation of the noncanonical NF-KB pathway. Int $\mathrm{J}$ Cancer. 2015;136(10):2341-2351.

34. Crawley CD, Kang S, Bernal GM, et al. S-phase-dependent p50/NF-KB1 phosphorylation in response to ATR and replication stress acts to maintain genomic stability. Cell Cycle. 2015;14(4):566-576.

35. Wang J, Jacob NK, Ladner KJ, et al. RelA/p65 functions to maintain cellular senescence by regulating genomic stability and DNA repair. EMBO Rep. 2009;10(11):1272-1278.

36. Fennell KA, Bell CC, Dawson MA. Epigenetic therapies in acute myeloid leukemia: where to from here? Blood.

2019;134(22):1891-1901.

37. Betzler AC, Theodoraki M-N, Schuler PJ, et al. NF-KB and Its role 
in checkpoint control. Int J Mol Sci. 2020;21(11):3949

38. Xia Y, Shen S, Verma IM. NF-KB, an active player in human cancers. Cancer Immunol Res. 2014;2(9):823-830.

39. Lu C, Klement JD, Smith AD, et al. p50 suppresses cytotoxic T lymphocyte effector function to regulate tumor immune escape and response to immunotherapy. J Immunother Cancer. 2020;8(2):e001365.

40. Borst J, Hendriks J, Xiao Y. CD27 and CD70 in T cell and B cell activation. Curr Opin Immunol. 2005;17(3):275-281.

41. Riether $C$, Schürch $C$, Ochsenbein AF. Modulating CD27 signaling to treat cancer. Oncoimmunology. 2012;1(9):1604-1606.

42. Židovec Lepej S, Matulić M, Gršković P, Pavlica M, Radmanić L, Korać P. miRNAs: EBV mechanism for escaping host's immune response and supporting tumorigenesis. Pathogens. 2020;9(5.):353

43. Wang L, Qin W, Huo Y-J, et al. Advances in targeted therapy for malignant lymphoma. Signal Transduct Target Ther. 2020;5(1):15.

44. Godwin P, Baird AM, Heavey S, Barr MP, O’Byrne KJ, Gately K. Targeting nuclear factor-kappa $B$ to overcome resistance to chemotherapy. Front Oncol. 2013;3:120.

45. Spaccarelli N, Rook AH. The use of interferons in the treatment of cutaneous T-cell lymphoma. Dermatol Clin.

2015;33(4):731-745.
46. Chihara N, Madi A, Kondo T, et al. Induction and transcriptional regulation of the co-inhibitory gene module in T cells. Nature. 2018;558(7710):454-459.

47. Green MR, Monti S, Rodig SJ, et al. Integrative analysis reveals selective 9p24.1 amplification, increased PD-1 ligand expression, and further induction via JAK2 in nodular sclerosing Hodgkin lymphoma and primary mediastinal large B-cell lymphoma. Blood. 2010;116(17):3268-3277.

48. Lim JQ, Huang D, Tang T, et al. Whole-genome sequencing identifies responders to pembrolizumab in relapse/refractory natural-killer/T cell lymphoma. Leukemia. 2020;34(12):3413-3419.

49. Xu ZG, Iwatsuki K, Oyama N, et al. The latency pattern of Epstein-Barr virus infection and viral IL-10 expression in cutaneous natural killer/T-cell lymphomas. Br J Cancer. 2001;84(7):920-925.

50. Chiang AK, Tao Q, Srivastava G, Ho FC. Nasal NK- and T-cell lymphomas share the same type of Epstein-Barr virus latency as nasopharyngeal carcinoma and Hodgkin's disease. Int J Cancer. 1996;68(3):285-290.

51. Yamashita D, Shimada K, Takata K, et al. Reappraisal of nodal Epstein-Barr virus-negative cytotoxic T-cell lymphoma: Identification of indolent CD5 diseases. Cancer Sci. 2018;109(8):2599-2610. 